This item was submitted to Loughborough's Research Repository by the author.

Items in Figshare are protected by copyright, with all rights reserved, unless otherwise indicated.

\title{
Exploiting diversity for optimizing margin distribution in ensemble learning
}

PLEASE CITE THE PUBLISHED VERSION

http://dx.doi.org/10.1016/j.knosys.2014.06.005

\section{PUBLISHER}

(c) Elsevier

\section{VERSION}

AM (Accepted Manuscript)

\section{PUBLISHER STATEMENT}

This work is made available according to the conditions of the Creative Commons Attribution-NonCommercialNoDerivatives 4.0 International (CC BY-NC-ND 4.0) licence. Full details of this licence are available at: https://creativecommons.org/licenses/by-nc-nd/4.0/

\section{LICENCE}

CC BY-NC-ND 4.0

\section{REPOSITORY RECORD}

Hu, Qinghua, Leijun Li, Xiangqian Wu, Gerald Schaefer, and Daren Yu. 2019. "Exploiting Diversity for Optimizing Margin Distribution in Ensemble Learning". figshare. https://hdl.handle.net/2134/25432. 


\title{
Exploiting Diversity for Optimizing Margin Distribution in Ensemble Learning
}

\author{
Qinghua $\mathrm{Hu}^{a}$, Leijun $\mathrm{Li}^{a}$, Xiangqian $\mathrm{Wu}^{a}$, Gerald Schaefer ${ }^{b}$ and Daren $\mathrm{Yu}^{c}$ \\ ${ }^{a}$ Biometric Computing Research Centre, School of Computer Science and Technology, \\ Harbin Institute of Technology, Harbin 150001, China \\ ${ }^{b}$ Department of Computer Science, Loughborough University, U.K. \\ ${ }^{c}$ School of Energy Science and Engineering, Harbin Institute of Technology, Harbin 150001, \\ China
}

\begin{abstract}
Margin distribution is acknowledged as an important factor for improving the generalization performance of classifiers. In this paper, we propose a novel ensemble learning algorithm named Double Rotation Margin Forest (DRMF), that aims to improve the margin distribution of the combined system over the training set. We utilise random rotation to produce diverse base classifiers, and optimize the margin distribution to exploit the diversity for producing an optimal ensemble. We demonstrate that diverse base classifiers are beneficial in deriving large-margin ensembles, and that therefore our proposed technique will lead to good generalization performance. We examine our method on an extensive set of benchmark classification tasks. The experimental results confirm that DRMF outperforms other classical ensemble algorithms such as Bagging, AdaBoostM1 and Rotation Forest. The success of DRMF is explained from the viewpoints of margin distribution and diversity.
\end{abstract}

Keywords: Ensemble learning, margin distribution, diversity, fusion strategy, rotation

\section{Introduction}

Ensemble learning has been an active research area in pattern recognition and machine learning domains for more than twenty years [1, 29, 38, 45, 59]. Ensemble learning, also referred to as multiple classifier systems, committees of learners, decision forest or consensus theory, is based on the idea of training a set of base classifiers or regressors for a given learning task and combining their outputs through a fusion strategy.

A significant amount of works have been focused on designing effective ensemble classifiers $[15,20,25,39]$. However, an exact explanation of the success

Email address: huqinghua@hit.edu.cn, lileijun1985@163.com, xqwu@hit.edu.cn, Gerald.Schaefer@ieee.org, yudaren@hit.edu.cn (Qinghua $\mathrm{Hu}^{a}$, Leijun $\mathrm{Li}^{a}$, Xiangqian $\mathrm{Wu}^{a}$, Gerald Schaefer ${ }^{b}$ and Daren $\mathrm{Yu}^{c}$ ) 
of ensemble strategies is still an open problem. Some researchers explored how an ensemble's effectiveness is related to the large margin principle, which is regarded as an important factor for improving classification [42, 51]. In this paper, we propose a novel ensemble learning algorithm named Double Rotation Margin Forest (DRMF), which is designed to improve the margin distribution of ensembles by enhancing the diversity of base classifiers and exploiting this diversity using an optimization technique.

In general, there are two well-accepted viewpoints - diversity and margin to explain the success of ensemble learning. Roughly speaking, the margin of a sample is its distance from the decision boundary and thus reflects the confidence of the classification. The margin distribution is acknowledged as an important factor for improving the generalization performance of classifiers $[2,6,11,43,49]$. In [43], Shawe-Taylor et al. gave an upper bound of generalization error in terms of the margin, while in [6] a similar bound was derived for neural networks with small weights. The large margin principle has been employed to design classification algorithms in $[8,14,21,26,50]$.

The performance of ensemble learning methods, especially boosting, has been attributed to the improvement of the margin distribution of the training set $[42,51]$. In AdaBoost, each new base classifier is trained by taking into account the performance of the previous base classifiers. Training samples that are misclassified by the current base classifiers play a more important role in training the subsequent one. The success of Adaboost can thus be explained from the margin distribution, where the optimization objective is to minimize a margin distribution based exponential loss function. In [42], an upper bound of the generalization error was derived in terms of the margins of the training samples, and it was shown that the generalization performance was determined by the margin distribution, the number of training samples and the number of base classifiers. The efficacy of AdaBoost thus lies in its ability of effectively improving the margin distribution. In [51], Wang et al. showed that a larger Equilibrium margin (Emargin) and a smaller Emargin error can reduce the generalization error, and demonstrated that AdaBoost can produce a larger Emargin and a smaller Emargin error.

It is acknowledged that the diversity among the members of an ensemble is crucial for performance improvement. Intuitively, no improvement can be achieved when a set of identical classifiers are combined. Diversity thus allows different classifiers to offer complementary information for classification, which in turn can lead to better performance [28]. A number of techniques have been proposed to introduce diversity. In general, we can divide these into two categories: classifier perturbation and sample perturbation approaches. Classifier perturbation refers to the adoption of instability of learning algorithms $[10,36]$ such as decision trees and neural networks. Since they are sensitive to initialization, trained predictors may converge to different local minima if started from different initializations, and diversity can thus be generated from trained classifiers. Sample perturbation techniques train classifiers on different sample subsets or feature subsets, and include bagging, boosting, random subspaces and similar approaches $[4,7,17,41]$. 
Since both diversity and margin are argued to explain the success of ensemble learning, it appears natural to question whether there is a connection between the two. Tang et al. [46] proved that maximizing the diversity among base classifiers is equivalent to optimizing the margin of an ensemble on the training samples if the average classification accuracy is constant and maximal diversity is achievable. Consequently, increasing the diversity among base classifiers is an effective method to improve the margin of ensembles. Our work is motivated by this conclusion, and our aim is to improve the margin distribution of ensembles.

In our proposed approach, we enhance the diversity of base classifiers by perturbing the samples using double random rotation. This idea is inspired by the PCA rotation proposed in the Rotation Forest algorithm [39]. In Rotation Forest, a candidate feature set is randomly split into $K$ subsets and Principal Component Analysis (PCA) is conducted on each subset to create diverse training samples. Diversity is thus promoted through the random feature splits for different base classifiers. In our work, the feature sets are also randomly split into $K$ subsets. In order to introduce further diversity between the base classifiers, we apply PCA and Locality Sensitive Discriminant Analysis (LSDA). In particular, we first perform unsupervised rotation with PCA, and then employ supervised large-margin rotation with LSDA. LSDA [12], as a supervised method, is able to derive a projection which maximizes the margin between data points from different classes. Our experimental results show that the applied Double Rotation can consistently enhance the diversity in a set of base classifiers.

We further exploit the diversity and improve the margin distribution with an optimal fusion strategy. In principle, there are two kinds of fusion strategies. One approach is to combine all available classifiers, e.g., in simple (plurality) voting (SV) [28] or through linear or non-linear combination rules [5, 9, 19, 48]. The other method is to derive selective ensembles, or pruned ensembles such as LP-Adaboost [23] or genetic algorithm (GA)-based approaches [53], which only select a fraction of the base classifiers for decision making and discard the others. Clearly, the key problem here is how to find an optimal subset of base classifiers [32]. In the GASEN approach [55], neural networks are selected based on evolved weights to constitute the ensemble. In [54], the subset selection problem is formulated as a quadratic integer programming problem, and semidefinite programming is adopted to select the base classifiers. Both GASEN and semi-definite programming are global optimization methods and thus their computational complexity is rather high. Suboptimal ensemble pruning methods were proposed to overcome this drawback, including reduce-error pruning [31], margin distance minimization (MDM) [33], orientation ordering [34], boostingbased ordering [35], and expectation propagation [13]. In practice, users would prefer sparse ensembles since computational resources are often limited [57]. In this paper, we introduce a technique to improve the margin distribution by minimizing the margin induced classification loss. In our pruned ensembles, the weights of base classifiers are trained with $L_{1}$ regularized squared loss [56]. The base classifiers are then sorted according to their weights, and those with large weights are selected in the final ensemble. 
Our presented work comprises three major contributions. First, since diversity is considered to be an important factor which affects the classification margin, Double Rotation is proposed to enhance the diversity among base classifiers. Second, we present a new pruned fusion method based on the Lasso technique for generating ensembles with optimal margin and sparse weight vectors, where the weights are learned through minimization of the regularized squared loss function. Third, we present an extensive set of experiments to evaluate the effectiveness and explain the rationality of the proposed algorithm. We convincingly show that it can improve the margin distribution to a great extent and lead to powerful ensembles.

The remainder of the paper is organized as follows. Related work is introduced in Section 2. Section 3 describes our proposed algorithm, while an analysis in terms of parameter sensitivity and robustness is presented in Section 4. Section 5 presents the experimental results and explores the rationality of DRMF. Finally, Section 6 offers conclusions and future work.

\section{Related Work}

Assume that $x_{i}=\left[x_{i 1}, \cdots, x_{i n}\right]^{T}$ is a sample represented by a set $F$ of $n$ features and every sample is generated independently at random according to some fixed but unknown distribution $\mathfrak{D}$. Let $X$ be an $N \times n$ matrix containing the training set and $Y=\left[y_{1}, \cdots, y_{N}\right]^{T}$ be an $N$-dimensional vector containing the class labels for the data, where $y_{i}$ is a class label of $x_{i}$ from the set of the class labels $\left\{\omega_{1}, \cdots, \omega_{c}\right\}$. Let $\left\{\mathcal{C}_{1}, \cdots, \mathcal{C}_{L}\right\}$ be the set of base classifiers in an ensemble. In this paper, our aim is to obtain an ensemble system with small generalization error via optimizing the margin distribution. Here, the generalization error of a classifier $\mathcal{C}_{j}$ is the probability of $\mathcal{C}_{j}(x) \neq y$ when an example $(x, y)$ is chosen at random according to the distribution $\mathfrak{D}$ and denoted as $P_{\mathfrak{D}}\left[\mathcal{C}_{j}(x) \neq y\right]$. The margin distribution is a function of $\theta$ which gives the fraction of samples whose margin is smaller than $\theta$. A good margin distribution means that most examples have large margins.

Definition 1. Given $x_{i} \in X, h_{i j}(j=1,2 \cdots, L)$ is the output of $x_{i}$ from $\mathcal{C}_{j}$ We define

$$
d_{i j}=\left\{\begin{array}{c}
1, \text { if } y_{i}=h_{i j} \\
-1, \text { if } y_{i} \neq h_{i j}
\end{array}\right.
$$

where $y_{i}$ is the real class label of $x_{i}$.

From this definition, we know that $d_{i j}=1$ if $x_{i}$ is correctly classified by $\mathcal{C}_{j}$; otherwise $d_{i j}=-1$.

Definition 2. [42] Given $x_{i} \in X$, the margin of $x_{i}$ in terms of the ensemble is defined as

$$
m\left(x_{i}\right)=\sum_{j=1}^{L} w_{j} d_{i j},
$$

where $w_{j}$ is the weight of $\mathcal{C}_{j}$ and $w_{j}>0$. 
In $[42,51]$, it is shown that a small generalization error for a voting classifier can be obtained by a good margin distribution on the training set. Obviously, the performances of the base classifiers have a significant effect on the margin of $x_{i}$. At the same time, the diversity among base classifiers is another key factor. In [46], the underlying relationship between diversity and margin was analyzed.

Theorem 1. [46] Let $\Theta$ be the average classification accuracy of the base classifiers. If $\Theta$ is regarded as a constant and if maximum diversity is achievable, maximization of the diversity among base classifiers is equivalent to maximization of the minimal margin of the ensemble on the training samples.

It should be noted that our aim is not to maximize the minimal margin of the ensemble, but to optimize the margin distribution. We use a disagreement measure [30] to measure the diversity of the base classifiers in our approach. The diversity between classifiers $\mathcal{C}_{j}$ and $\mathcal{C}_{k}$ is thus computed as

$$
D i s_{j k}=\frac{N^{01}+N^{10}}{N^{11}+N^{10}+N^{01}+N^{00}},
$$

where $N^{00}$ denotes the number of samples misclassified by both classifiers, $N^{11}$ is the number of samples correctly classified by both, $N^{10}$ denotes the number of samples which were correctly classified by $\mathcal{C}_{j}$ but misclassified by $\mathcal{C}_{k}$, and $N^{01}$ denotes the number of samples misclassified by $\mathcal{C}_{j}$ but correctly classified by $\mathcal{C}_{k}$. For multiple base classifiers, the overall diversity is computed as the average diversity of classifier pairs.

In [39], Rodríguez and Kuncheva designed a method to generate ensembles based on feature transformation. The diversity of base classifiers is promoted by random splits of the feature set into different subsets. The original feature space is split into $K$ subspaces (the subsets may be disjoint or may intersect). Then, PCA is applied to linearly rotate the subspaces along the "rotation" matrix. Diversity is obtained by random splits of the feature set.

Cai et al. [12] proposed a supervised algorithm for feature transformation, which can find a projection that maximizes the margin between different classes. For $x_{i} \in X$, denote by $\Upsilon\left(x_{i}\right)=\left\{x_{i}^{1}, \cdots, x_{i}^{e}\right\}$ the set of its $e$ nearest neighbors and by $y_{i}$ the class label of $x_{i}$. We define

$$
\Upsilon_{s}\left(x_{i}\right)=\left\{x_{i}^{j} \mid y_{i}^{j}=y_{i}, 1 \leq j \leq e\right\},
$$

and

$$
\Upsilon_{b}\left(x_{i}\right)=\left\{x_{i}^{j} \mid y_{i}^{j} \neq y_{i}, 1 \leq j \leq e\right\},
$$

so that $\Upsilon_{s}\left(x_{i}\right)$ contains the neighbors which share the same label with $x_{i}$, while $\Upsilon_{b}\left(x_{i}\right)$ is the set of the neighbors which belong to the other classes.

For any $x_{i}$ and $x_{j}$, we define

$$
V_{b, i j}=\left\{\begin{array}{l}
1 \text { if } x_{i} \in \Upsilon_{b}\left(x_{j}\right) \text { or } x_{j} \in \Upsilon_{b}\left(x_{i}\right) \\
0 \text { otherwise }
\end{array},\right.
$$


and

$$
V_{s, i j}=\left\{\begin{array}{l}
1 \text { if } x_{i} \in \Upsilon_{s}\left(x_{j}\right) \text { or } x_{j} \in \Upsilon_{s}\left(x_{i}\right) . \\
0 \text { otherwise }
\end{array}\right.
$$

$V_{b}$ and $V_{s}$ thus give the weight matrices of the between-class graph $G_{b}$ and the within-class graph $G_{s}$ respectively.

The objective of Locality Sensitive Discriminant Analysis (LSDA) is to map the within-class graph and the between-class graph to a line so that the connected points of $G_{s}$ stay as close as possible while the connected points of $G_{b}$ are as distant as possible. Suppose $x_{1}, \cdots, x_{N}$ are mapped to $z_{1}, \cdots, z_{N}$ and $z_{i}=\vartheta^{T} x_{i}$ where $\vartheta$ is a projection vector. In order to compute $z_{1}, \cdots, z_{N}$, the following Locality Sensitive Discriminant (LSD) objective functions are optimized:

$$
\begin{aligned}
& \min \sum_{i j}\left(z_{i}-z_{j}\right)^{2} V_{s, i j}, \\
& \max \sum_{i j}\left(z_{i}-z_{j}\right)^{2} V_{b, i j} .
\end{aligned}
$$

This optimization can be translated into maximum eigenvalue solutions to the generalized eigenvalue problem

$$
X^{T}\left(p \Lambda_{b}+(1-p) V_{s}\right) X \vartheta=\lambda X^{T} Q_{s} X \vartheta,
$$

where $X$ is an $N \times n$ matrix, $Q_{s}$ is a diagonal matrix whose entries are the column sums of $V_{s}$, and $\Lambda_{b}=Q_{b}-V_{b}$ where $Q_{b}$ is a diagonal matrix whose entries are column sums of $V_{b}$. From the LSD objective functions, it can be seen that LSDA can discover both geometrical and discriminant structures in the data.

\section{Algorithm Description}

As shown above, diversity is an important factor to improve margin distribution. In $[42,51]$, the relationship between the generalization performance and the margin distribution of the training set was derived. It was found that if a voting classifier generates a good margin distribution, the generalization error will be small. Motivated by these results, we propose a novel technique to generate diverse base classifiers and to exploit diversity for producing good ensembles with an optimal margin distribution.

\subsection{Double Rotation}

Double Rotation aims to enhance the diversity among base classifiers. In order to construct the training set for the base classifier $\mathcal{C}_{j}$, we first split the feature set $F$ randomly into $K$ subsets $F_{i j}(i=1,2 \cdots, K)$ which contain $M=$ $\llcorner n / K\lrcorner$ features ( $\llcorner n / K\lrcorner$ rounds $n / K$ to the nearest integer) and denote by $X_{i j}$ the data subset with features $F_{i j}$. We then eliminate a random subset of the classes and draw $\gamma \cdot N$ samples by bootstrapping from $X_{i j}$ to obtain a new set 
$X_{i j}^{\prime}$. We apply PCA on $X_{i j}^{\prime}$ to obtain the coefficients of the principal components $\mathrm{a}_{i, j}^{1}, \cdots, \mathrm{a}_{i, j}^{M_{i} 1}$. From these we construct a "rotation" matrix $R_{j}$

$$
R_{j}=\left[\begin{array}{cccc}
\mathrm{a}_{1, j}^{1}, \cdots, \mathrm{a}_{1, j}^{M_{1}} & 0 & \cdots & 0 \\
0 & \mathrm{a}_{2, j}^{1}, \cdots, \mathrm{a}_{2, j}^{M_{2}} & \cdots & 0 \\
0 & 0 & \ddots & 0 \\
0 & 0 & \cdots & \mathrm{a}_{K, j}^{1}, \cdots, \mathrm{a}_{K, j}^{M_{K}}
\end{array}\right] .
$$

The columns of $R_{j}$ are rearranged so that they correspond to the original features. If we denote the rearranged rotation matrix as $R_{j}^{a}$, then $X R_{j}^{a}$ is taken as a new training set. We then repeat the above process but replace PCA with LSDA and obtain a new rotation matrix $S_{j}^{a}$. Finally, $\mathcal{C}_{j}$ is trained with $\left(X R_{j}^{a} S_{j}^{a}, Y\right)$.

The pseudocode of the Double Rotation algorithm is formulated in Algorithm 1.

Double Rotation integrates two different feature transformation algorithms for boosting the diversity among base classifiers. In DRMF, the base classifiers are trained with the data $\left(X R_{j}^{a} S_{j}^{a}, Y\right)$ based on the J48 algorithm, an implementation of C4.5 in the WEKA library [27].

\subsection{Ensemble Pruning by optimizing margin distribution}

Based on the above procedure, we obtain a set of diverse decision tree classifiers. Now, we exploit this diversity to construct an optimal ensemble.

Given $x \in X, h_{x j} \in\{-1,1\}$ as the output of $x$ from $\mathcal{C}_{j}$, and $w_{j}$ as the weight of $\mathcal{C}_{j}$, the final decision function is

$$
f(x)=\operatorname{sgn}\left(\sum_{j=1}^{L} w_{j} h_{x j}\right) .
$$

Here, $f(x)$ can be seen as a linear classifier in a new input space, where every sample $x$ is represented as an $L$-dimensional vector $\left[h_{x 1}, \cdots, h_{x L}\right]_{L \times 1}^{T}$, and then $w_{j}(j=1,2, \cdots, L)$ can be seen as the coefficients of this function. Based on the conclusion in [44], a bound of the generalization error for the linear classifier can be derived as follows.

Theorem 2. For $\Delta>0, t \in \Re$, consider a fixed but unknown probability distribution on the input space $\Phi$ with support in the ball of radius $\mathbb{R}$ about the origin. Then, with probability $1-\delta$ over randomly drawn training set $X$ of size $N$ for all $\beta>0$ the generalization of the linear classifier $f(x)$ on the input space is bounded by

$$
\epsilon(N, \eta, \delta)=\frac{2}{N}\left(\eta \log _{2}\left(\frac{8 e N}{\eta}\right) \log _{2}(32 N)+\log _{2}\left(\frac{8 N}{\delta}\right)\right),
$$

\footnotetext{
${ }^{1}$ The reason for eliminating a random subset of classes and drawing $\gamma \cdot N$ samples by bootstrapping is to avoid identical coefficients of principal components when the same feature subset is chosen for different classifiers.
} 


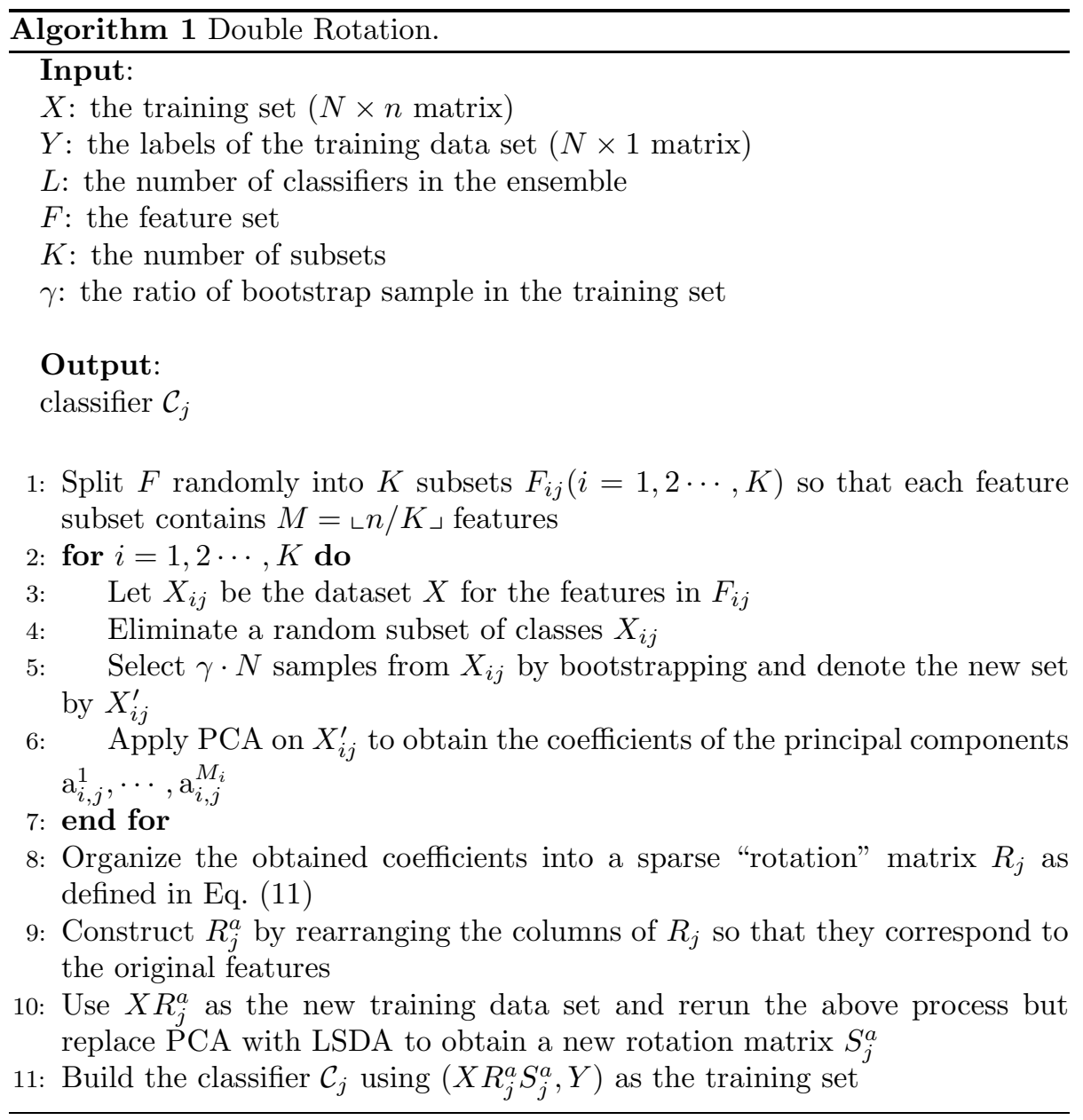

where

$$
\eta=\left\lfloor\frac{64.5\left(\mathbb{R}^{2}+\Delta^{2}\right)\left(\|W\|^{2}+E(X,(W, t), \beta)^{2} / \Delta^{2}\right)}{\beta^{2}}\right\rfloor,
$$

provided $N \geq 2 / \epsilon$, and $\eta \leq e N$.

In Theorem $2, W=\left[w_{1}, \cdots, w_{L}\right]_{L \times 1}^{T}, x=\left[h_{x 1}, \cdots, h_{x L}\right]_{L \times 1}^{T}, y$ is the real class label of $x$ and $t=0$. Besides, $E(X,(W, t), \beta)=\sqrt{\sum_{(x, y) \in X} \varphi((x, y),(W, t), \beta)^{2}}$ and $\varphi((x, y),(W, t), \beta)=\max \left\{0, \beta-y\left(\left\langle W^{T} \cdot x\right\rangle-t\right)\right\}$. We can see that with $\beta$ given, a small $\mathbb{R}$ and $E(X,(W, t), \beta)$ can lead to a good linear classifier. In fact, $\mathbb{R}$ is related to the number of base classifiers in the ensemble since if there are fewer base classifiers, $\mathbb{R}$ will become smaller. On the other hand, $y\left(\left\langle W^{T} \cdot x\right\rangle-t\right)=y\left(\left\langle W^{T} \cdot x\right\rangle\right)=y\left(\sum_{j=1}^{L} w_{j} h_{x j}\right)=\sum_{j=1}^{L} w_{j} y h_{x j}=\sum_{j=1}^{L} w_{j} d_{x j}=m(x)$ 
when $t=0$ and $y, h_{x j} \in\{-1,1\}$. Thus, $E(X,(W, t), \beta)$ can be understood as the root of the squared loss of ensembles, which is determined by the margin distribution of the ensemble. Based on the theorem, we can design an optimization objective to learn the weights of the base classifiers.

Definition 3. Given $x_{i} \in X$, the classification loss of $x_{i}$ is computed as

$$
l\left(x_{i}\right)=\left[1-m\left(x_{i}\right)\right]^{2},
$$

where $m\left(x_{i}\right)$ is the margin of $x_{i}$. Then, the classification loss of $X$ is

$$
l(X)=\sum_{i=1}^{n} l\left(x_{i}\right)=\|U-D W\|_{2}^{2},
$$

where $U=[1, \cdots, 1]_{N \times 1}^{T}, W=\left[w_{1}, \cdots, w_{L}\right]_{L \times 1}^{T}$ and $D=\left\{d_{i j}\right\}_{N \times L}$.

The above function only considers the classification loss related to the margin distribution. The number of base classifiers is not taken into account. However, from Theorem 2 we know that only few base classifiers should be included in ensembles, and consequently the weight vector should be sparse. A sparse model is expected to improve the generalization performance $[18,22,58]$. In order to obtain a sparse weight vector, we add the $L_{1}$ norm regularization term of the weight vector into the loss function. The regularized loss function is then

$$
J_{W}=\operatorname{argmin}\|U-D W\|_{2}^{2}+\lambda\|W\|_{1} .
$$

This is the well-known Lasso problem [47]. While Lasso has been employed in regression ensembles [24], we here apply it to classification tasks. Lasso can be explained as a large margin solution in classification in terms of the infinite norm [40]. By minimizing $J_{W}$, we obtain the weights $w_{j}(j=1,2, \cdots, L)$ of the base classifiers.

Given the weight coefficients, the base classifiers are sorted, and then a suboptimal subset is selected for classifying previously unseen samples. Algorithm 2 describes the approach in pseudocode.

Essentially, our proposed technique is an ordered aggregation pruning method based on the weights of the base classifiers, where the weights are trained by minimizing a margin induced classification loss.

\section{Algorithm analysis}

There are several parameters to be set in our proposed algorithm. In this section, we discuss how these parameters affect the performance of the generated classifier.

First, we discuss how to set $K$, i.e. the number of splits. We do this based on numerical experiments on various UCI [3] datasets. We set $K$ to $2,3,4,5$, and then compare the resulting performances of DRMF. The ratio of bootstrap 


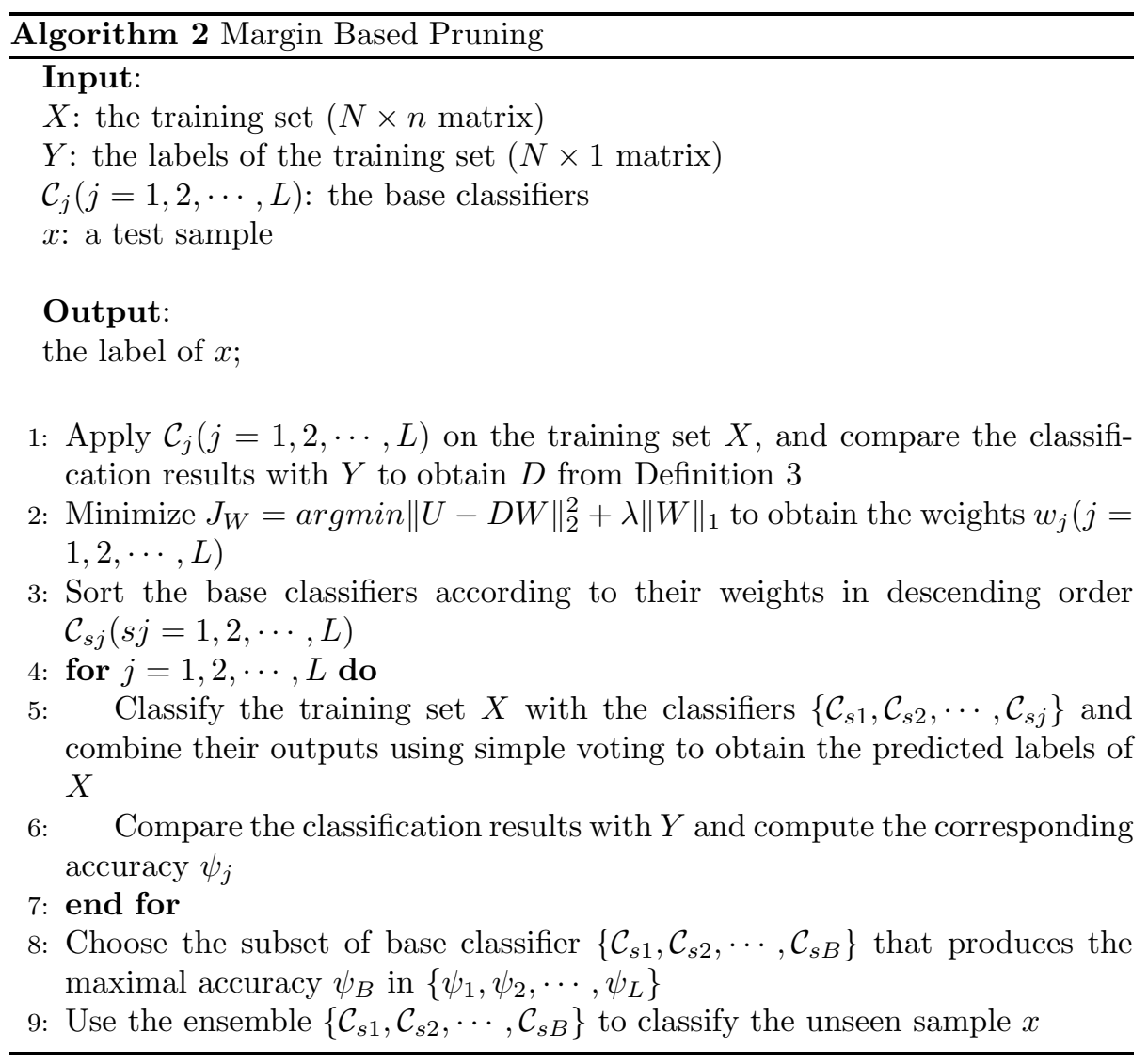

samples was set to 0.75 , the number of candidate base classifiers was 100 and J48 decision trees from the WEKA library [27] were used as base classifiers.

Table 1 describes the 20 classification tasks we used. For every classification task and data set, standard 10-fold cross validation was performed.

In Table 2, we report the classification performance of DRMF with different random splits. As we can see from there, $K$ has some influence on the performance of the generated ensembles. Since random splits of the features lead to different rotations, diverse base classifiers are generated. However, if $K$ is too large, the number of features in each subset may become too small and hence not sufficient to effectively represent the learning task, thus leading to a drop in performance. Clearly, a tradeoff between the diversity and the performances of the base classifiers should be made. The experimental results in Table 2 indicate that DRMF produces good performance if $K=2$, and we consequently set $K=2$ in the remainder of experiments.

In DRMF, the number of candidate base classifiers should be set before training. Hence, next we investigate how many candidate base classifiers are sufficient to lead to a good ensemble. We thus perform experiments varying the 
Table 1: Statistics of classification tasks.

\begin{tabular}{|c|c|c|c|c|}
\hline Dataset & Instances & Discrete Features & Continuous Features & Classes \\
\hline \hline australian & 690 & 8 & 6 & 2 \\
\hline crx & 690 & 9 & 6 & 2 \\
\hline cmc & 1473 & 7 & 2 & 3 \\
\hline derm & 366 & 0 & 34 & 6 \\
\hline german & 1000 & 13 & 7 & 2 \\
\hline glass & 214 & 0 & 9 & 6 \\
\hline heart & 270 & 0 & 13 & 2 \\
\hline horse & 368 & 15 & 7 & 2 \\
\hline ICU & 200 & 16 & 4 & 3 \\
\hline iono & 351 & 0 & 34 & 2 \\
\hline iris & 150 & 0 & 4 & 3 \\
\hline movement & 360 & 0 & 90 & 15 \\
\hline pima & 768 & 0 & 8 & 2 \\
\hline rice & 104 & 0 & 5 & 2 \\
\hline spectf & 269 & 0 & 44 & 2 \\
\hline thyroid & 215 & 0 & 5 & 3 \\
\hline wiscon & 699 & 0 & 9 & 2 \\
\hline wdbc & 569 & 0 & 30 & 2 \\
\hline yeast & 1484 & 0 & 7 & 2 \\
\hline zoo & 101 & 15 & 1 & 7 \\
\hline
\end{tabular}

Table 2: Classification performance of DRMF with different numbers of splits.

\begin{tabular}{|c|c|c|c|c|}
\hline Data set & $\mathrm{K}=2$ & $\mathrm{~K}=3$ & $\mathrm{~K}=4$ & $\mathrm{~K}=5$ \\
\hline \hline australian & $\mathbf{8 8 . 1 1} \pm \mathbf{3 . 4 8}$ & $87.24 \pm 4.10$ & $87.10 \pm 2.94$ & $87.97 \pm 3.63$ \\
\hline crx & $86.37 \pm 13.66$ & $86.22 \pm 15.01$ & $\mathbf{8 6 . 5 3} \pm \mathbf{1 3 . 5 8}$ & $86.37 \pm 14.28$ \\
\hline cmc & $54.24 \pm 3.25$ & $54.45 \pm 3.75$ & $\mathbf{5 5 . 5 4} \pm \mathbf{3 . 4 3}$ & $52.89 \pm 2.65$ \\
\hline derm & $\mathbf{9 6 . 7 5} \pm \mathbf{3 . 8 4}$ & $95.95 \pm 4.28$ & $96.19 \pm 3.22$ & $95.71 \pm 3.20$ \\
\hline german & $\mathbf{7 7 . 8 0} \pm \mathbf{2 . 9 4}$ & $76.70 \pm 3.33$ & $75.60 \pm 3.63$ & $76.40 \pm 4.01$ \\
\hline glass & $\mathbf{7 6 . 6 4} \pm \mathbf{1 0 . 6 1}$ & $73.37 \pm 8.64$ & $75.69 \pm 11.01$ & $62.06 \pm 14.65$ \\
\hline heart & $84.44 \pm 4.88$ & $83.70 \pm 5.00$ & $\mathbf{8 6 . 3 0} \pm \mathbf{3 . 5 1}$ & $80.00 \pm 6.34$ \\
\hline horse & $\mathbf{9 3 . 4 9} \pm \mathbf{3 . 8 3}$ & $91.85 \pm 5.25$ & $92.94 \pm 4.06$ & $93.21 \pm 3.88$ \\
\hline ICU & $93.56 \pm 4.80$ & $89.45 \pm 11.53$ & $\mathbf{9 3 . 6 1} \pm \mathbf{3 . 9 9}$ & $90.98 \pm 8.51$ \\
\hline iono & $93.47 \pm 4.76$ & $\mathbf{9 5 . 2 4} \pm \mathbf{3 . 6 9}$ & $93.52 \pm 5.05$ & $95.21 \pm 4.56$ \\
\hline iris & $\mathbf{9 8 . 6 7} \pm \mathbf{2 . 8 1}$ & $94.00 \pm 4.92$ & $96.00 \pm 3.44$ & $96.00 \pm 3.44$ \\
\hline movement & $\mathbf{8 2 . 4 4} \pm \mathbf{1 6 . 2 9}$ & $81.56 \pm 17.95$ & $81.00 \pm 16.63$ & $80.22 \pm 19.09$ \\
\hline pima & $\mathbf{7 8 . 7 8} \pm \mathbf{3 . 7 6}$ & $77.35 \pm 4.90$ & $77.87 \pm 4.61$ & $73.57 \pm 3.65$ \\
\hline rice & $\mathbf{8 9 . 8 2} \pm \mathbf{1 3 . 1 7}$ & $79.05 \pm 10.04$ & $79.96 \pm 10.70$ & $79.05 \pm 10.04$ \\
\hline spectf & $82.48 \pm 7.91$ & $83.28 \pm 3.08$ & $82.58 \pm 7.00$ & $\mathbf{8 3 . 9 4} \pm \mathbf{7 . 6 3}$ \\
\hline thyroid & $\mathbf{9 6 . 2 6} \pm \mathbf{4 . 8 6}$ & $93.48 \pm 7.92$ & $93.96 \pm 8.17$ & $93.48 \pm 5.93$ \\
\hline wiscon & $\mathbf{9 7 . 8 6} \pm \mathbf{2 . 3 6}$ & $97.57 \pm 3.16$ & $96.57 \pm 3.24$ & $92.99 \pm 3.71$ \\
\hline wdbc & $\mathbf{9 7 . 7 2} \pm \mathbf{1 . 6 6}$ & $95.80 \pm 3.42$ & $97.21 \pm 2.49$ & $96.84 \pm 2.58$ \\
\hline yeast & $\mathbf{7 3 . 2 5} \pm \mathbf{3 . 4 7}$ & $72.57 \pm 3.73$ & $70.68 \pm 5.80$ & $70.68 \pm 5.80$ \\
\hline zoo & $\mathbf{9 4 . 3 9} \pm \mathbf{8 . 3 9}$ & $92.39 \pm 11.40$ & $93.14 \pm 9.63$ & $\mathbf{9 4 . 3 9} \pm \mathbf{8 . 3 9}$ \\
\hline \hline Average & 86.83 & 85.06 & 85.60 & 84.10 \\
\hline
\end{tabular}


Table 3: Classification performance with different numbers of candidate base classifiers.

\begin{tabular}{|c|c|c|c|c|c|}
\hline Data set & $\mathrm{L}=20$ & $\mathrm{~L}=40$ & $\mathrm{~L}=60$ & $\mathrm{~L}=80$ & $\mathrm{~L}=100$ \\
\hline \hline australian & $86.96 \pm 3.01$ & $88.13 \pm 3.98$ & $87.54 \pm 2.90$ & $87.97 \pm 3.27$ & $88.11 \pm 3.48$ \\
\hline crx & $85.66 \pm 14.10$ & $85.51 \pm 14.66$ & $85.64 \pm 15.10$ & $86.81 \pm 13.20$ & $86.37 \pm 13.66$ \\
\hline cmc & $52.82 \pm 3.41$ & $53.97 \pm 3.30$ & $53.70 \pm 3.28$ & $54.18 \pm 2.95$ & $54.24 \pm 3.25$ \\
\hline derm & $96.47 \pm 3.90$ & $96.98 \pm 4.24$ & $95.91 \pm 5.26$ & $96.47 \pm 4.12$ & $96.75 \pm 3.84$ \\
\hline german & $75.40 \pm 3.60$ & $77.00 \pm 3.40$ & $77.00 \pm 3.02$ & $77.70 \pm 2.45$ & $77.80 \pm 2.94$ \\
\hline glass & $72.44 \pm 12.14$ & $74.44 \pm 11.43$ & $74.89 \pm 13.25$ & $78.14 \pm 10.91$ & $76.64 \pm 10.61$ \\
\hline heart & $83.33 \pm 3.60$ & $83.70 \pm 4.68$ & $84.81 \pm 4.43$ & $84.81 \pm 4.77$ & $84.44 \pm 4.88$ \\
\hline horse & $92.95 \pm 4.06$ & $92.94 \pm 4.06$ & $93.49 \pm 3.38$ & $93.22 \pm 3.39$ & $93.49 \pm 3.83$ \\
\hline ICU & $94.04 \pm 4.69$ & $94.04 \pm 4.69$ & $93.56 \pm 4.80$ & $94.09 \pm 5.21$ & $93.56 \pm 4.80$ \\
\hline iono & $93.20 \pm 4.00$ & $92.92 \pm 4.60$ & $93.49 \pm 4.95$ & $93.77 \pm 5.09$ & $93.47 \pm 4.76$ \\
\hline iris & $94.67 \pm 5.26$ & $94.67 \pm 5.26$ & $94.67 \pm 5.26$ & $96.67 \pm 4.71$ & $98.67 \pm 2.81$ \\
\hline movement & $80.56 \pm 15.15$ & $82.78 \pm 16.50$ & $82.78 \pm 16.57$ & $82.44 \pm 16.29$ & $82.44 \pm 16.29$ \\
\hline pima & $77.87 \pm 4.98$ & $78.52 \pm 3.90$ & $78.39 \pm 4.53$ & $78.39 \pm 4.32$ & $78.78 \pm 3.76$ \\
\hline rice & $89.73 \pm 10.18$ & $88.82 \pm 10.46$ & $90.73 \pm 12.86$ & $89.82 \pm 13.17$ & $89.82 \pm 13.17$ \\
\hline spectf & $82.61 \pm 5.18$ & $83.34 \pm 4.39$ & $82.12 \pm 7.21$ & $83.25 \pm 7.01$ & $82.48 \pm 7.91$ \\
\hline thyroid & $94.83 \pm 6.10$ & $95.30 \pm 6.31$ & $94.83 \pm 5.21$ & $95.78 \pm 5.19$ & $96.26 \pm 4.86$ \\
\hline wiscon & $97.34 \pm 2.59$ & $97.43 \pm 2.59$ & $97.71 \pm 2.15$ & $97.34 \pm 2.59$ & $97.86 \pm 2.36$ \\
\hline wdbc & $97.19 \pm 2.06$ & $97.72 \pm 1.66$ & $98.43 \pm 1.53$ & $97.72 \pm 1.66$ & $97.72 \pm 1.66$ \\
\hline yeast & $73.11 \pm 3.26$ & $73.45 \pm 3.12$ & $73.45 \pm 3.64$ & $73.38 \pm 3.61$ & $73.25 \pm 3.47$ \\
\hline zoo & $94.39 \pm 8.39$ & $94.39 \pm 8.39$ & $94.39 \pm 8.39$ & $94.39 \pm 8.39$ & $94.39 \pm 8.39$ \\
\hline \hline Average & 85.78 & 86.30 & 86.38 & 86.82 & 86.83 \\
\hline
\end{tabular}

number of base classifiers $L$ as 20,40,60, 80 and 100, respectively. Table 3 compares the classification performance of DRMF with these settings for $L$. As we can see from there, the overall performance improves when $L$ becomes larger. However, if $L \geqslant 80$, the difference is not significant, and we consequently use $L=100$ in the following experiments.

In Margin Based Pruning (Algorithm 2), we utilize simple voting in lines 5 and 9 . That is, the class that receives the largest number of votes is considered as the final decision. In contrast, in weighted voting, the votes are weighted and the ensemble decision is the class with the largest sum of weights of votes. Since we calculate the weights in line 2 of Algorithm 2, a natural question that arises is whether applying a weighted voting strategy would give better results. To answer this, we give, in Table 4, the classification accuracies and the number of selected base classifiers ${ }^{2}$ using the two fusion strategies. From Table 4 we can observe that simple voting performs typically better than weighted voting, while it also leads to smaller ensembles.

To further investigate why simple voting is better than weighted voting, we perform some additional experiments to explore the relationship between the weights of the base classifiers and their classification performances. Fig. 1 shows the relationship between the classification accuracy and the ranking of

${ }^{2}$ Different fusion strategies will lead to different ensemble sizes. 
Table 4: Classification performance and number of selected base classifiers for pruned simple voting (PSV) and pruned weighted voting (PWV).

\begin{tabular}{|c|c|c|c|c|}
\hline Data set & PSV accuracy & PSV no. class. & PWV accuracy & PWV no. class. \\
\hline \hline australian & $88.11 \pm 3.48$ & 13.9 & $87.10 \pm 3.83$ & 71.4 \\
\hline crx & $86.37 \pm 13.66$ & 18.2 & $85.50 \pm 12.22$ & 80.6 \\
\hline cmc & $54.24 \pm 3.25$ & 26.9 & $52.55 \pm 3.07$ & 81.7 \\
\hline derm & $96.75 \pm 3.84$ & 4.2 & $96.47 \pm 5.38$ & 3.7 \\
\hline german & $77.80 \pm 2.94$ & 20.6 & $76.70 \pm 3.43$ & 72.6 \\
\hline glass & $76.64 \pm 10.61$ & 9.8 & $78.51 \pm 7.88$ & 39.8 \\
\hline heart & $84.44 \pm 4.88$ & 15.4 & $82.59 \pm 4.95$ & 25.5 \\
\hline horse & $93.49 \pm 3.83$ & 4.4 & $91.03 \pm 4.24$ & 3.7 \\
\hline ICU & $93.56 \pm 4.80$ & 2.4 & $94.14 \pm 4.35$ & 11.9 \\
\hline iono & $93.47 \pm 4.76$ & 4.9 & $90.95 \pm 6.61$ & 2.6 \\
\hline iris & $98.67 \pm 2.81$ & 6.6 & $98.00 \pm 3.22$ & 1 \\
\hline movement & $82.44 \pm 16.29$ & 11.5 & $79.33 \pm 20.35$ & 7.2 \\
\hline pima & $78.78 \pm 3.76$ & 17.2 & $77.35 \pm 4.44$ & 72.7 \\
\hline rice & $89.82 \pm 13.17$ & 4.8 & $84.98 \pm 12.79$ & 51.4 \\
\hline spectf & $82.48 \pm 7.91$ & 4.9 & $80.24 \pm 8.33$ & 2.6 \\
\hline thyroid & $96.26 \pm 4.86$ & 1.8 & $95.78 \pm 5.19$ & 1.2 \\
\hline wiscon & $97.86 \pm 2.36$ & 4.4 & $97.86 \pm 2.54$ & 70 \\
\hline wdbc & $97.72 \pm 1.66$ & 8.5 & $97.01 \pm 2.05$ & 3.9 \\
\hline yeast & $73.25 \pm 3.47$ & 24.8 & $71.69 \pm 3.89$ & 70.6 \\
\hline zoo & $94.39 \pm 8.39$ & 1.3 & $93.39 \pm 8.24$ & 1 \\
\hline \hline Average & 86.83 & 10.33 & 85.56 & 33.76 \\
\hline
\end{tabular}

their weights. Here, the $\mathrm{x}$-axis indicates the ranking of the weight with 1 indicating the largest weight of the base classifier and 100 the smallest. From Fig. 1 we can conclude that base classifiers with large weights do not necessarily have better classification performance compared to those with small weights. Weight learning considers both the diversity among base classifiers and their performances and hence while the weights can be used to rank the base classifiers, they do not reflect their classification performances.

In our algorithm, the base classifiers are added to the ensemble one by one. Base classifiers with the large weights are included first. Fig. 2 plots the classification accuracies when the base classifiers are added, for both simple and weighted voting. From there, we can notice that the best classification accuracy obtained by simple voting is often higher than that of weighted voting, confirming that simple voting can produce better performance than weighted voting. We can also see that fusion based on only a subset of base classifiers is better than combining all of them, which is consistent with the findings from [55].

Margin Based Pruning has two main components. One is to learn the weights for the base classifiers via the minimization of $J_{W}$, while the other is to select the base classifiers. Some of the weights might be zero, and we consequently tested whether the classification performance is affected if we remove base classifier with zero weights. For this, in Table 5, we compare the fusion accuracy of base classifiers that receive non-zero weights (denoted by LASSO in Table 5) 

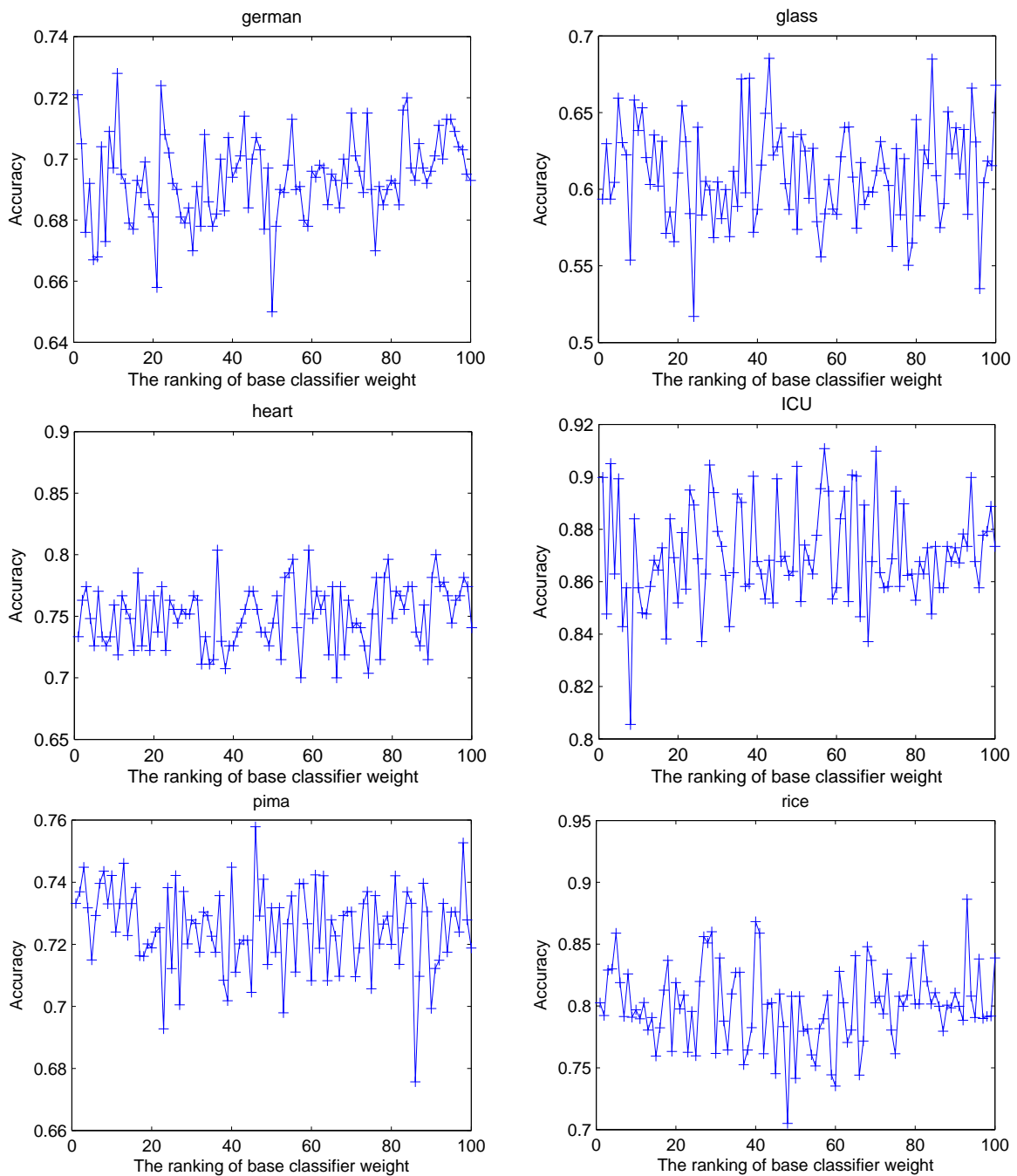

Figure 1: Classification accuracies of base classifiers with different ranking in terms of their weights.

with the fusion accuracy of DRMF. It can be seen that DRMF performs better than LASSO, and thus that Steps 3 to 8 in Algorithm 2 are indeed useful for improving the classification performance. We also analyze the differences between the base classifiers selected by the two strategies. From Table 5 it is apparent that the average accuracy of the base classifiers in DRMF is higher than that of the base classifiers that receive non-zero weights, and the diversity among base classifiers in DRMF is also higher than that of the base classifiers with non-zero weights.

Finally, we compare the robustness of DRMF with that of Rotation Forest 

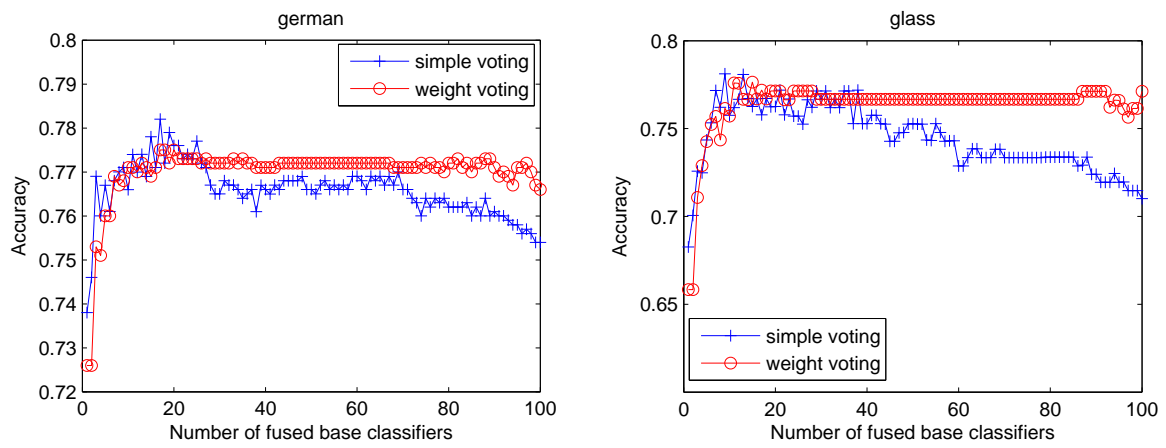

heart
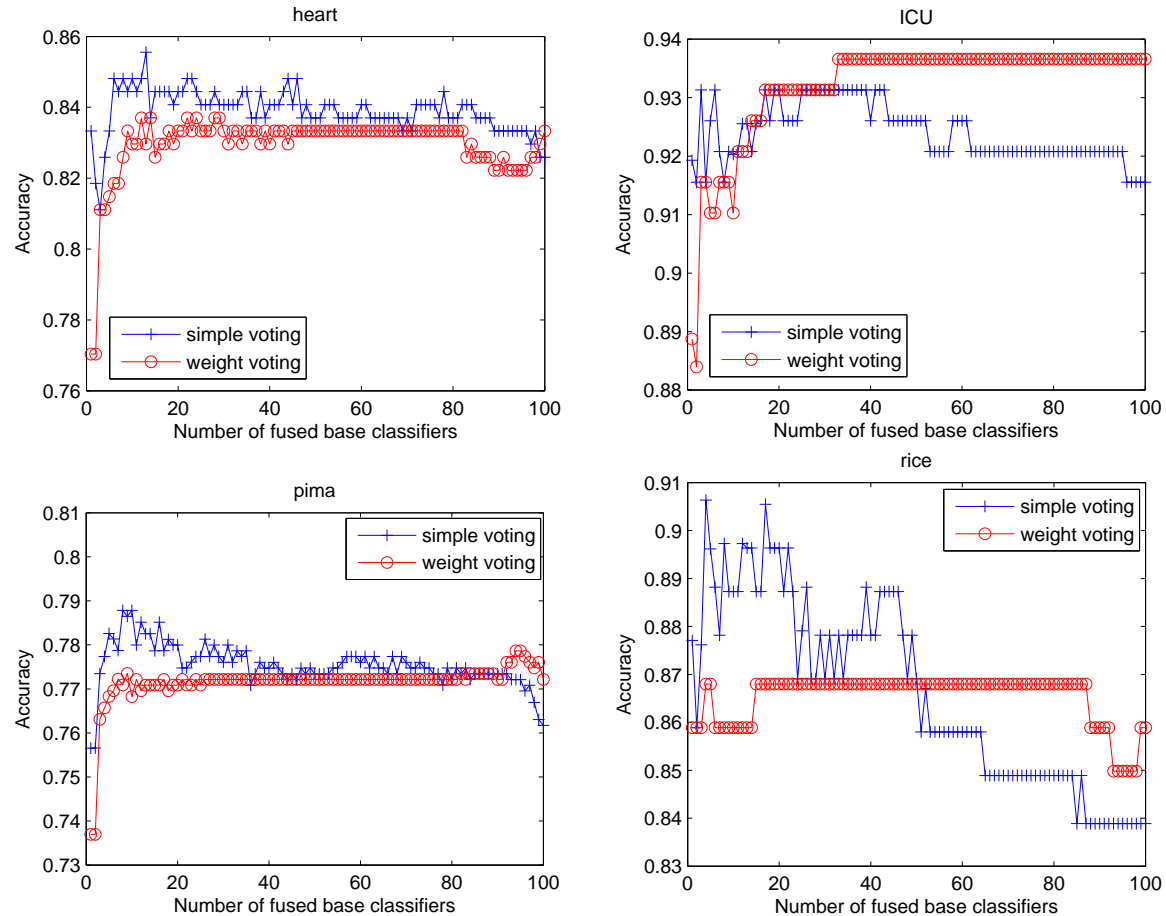

Figure 2: Variation of classification accuracies with different numbers of selected base classifiers when simple voting and weighted voting are used.

and J48. For that, we first generate noisy samples by randomly revising the labels of some training samples with the percentage of relabeled samples varying from $3 \%$ to $30 \%$. Fig. 3 shows the variation of classification accuracies when we increase the noise rate in the training set. As is apparent, DRMF shows superior robustness compared to both Rotation Forest and J48. In particular, for DRMF the variation of the classification accuracies remains small as the rate of mislabeled samples increases.

We further consider the robustness of the algorithms with respect to attribute noise and add Gaussian noise to the features of the training data. The 
Table 5: Comparison of different strategies used in selecting base classifiers (FA=fusion accuracy, AA=average accuracy).

\begin{tabular}{|c|c|c|c|c|c|c|}
\hline $\begin{array}{c}\text { Data } \\
\text { set }\end{array}$ & $\begin{array}{c}\text { FA } \\
(\text { LASSO })\end{array}$ & $\begin{array}{c}\text { FA } \\
(\text { DRMF })\end{array}$ & $\begin{array}{c}\text { Diversity } \\
(\text { LASSO })\end{array}$ & $\begin{array}{c}\text { Diversity } \\
(\text { DRMF })\end{array}$ & $\begin{array}{c}\text { AA } \\
\text { LASSO })\end{array}$ & $\begin{array}{c}\text { AA } \\
(\text { DRM })\end{array}$ \\
\hline \hline australian & $86.09 \pm 3.88$ & $88.11 \pm 3.48$ & 0.1122 & 0.1318 & 83.73 & 84.22 \\
\hline crx & $83.61 \pm 18.39$ & $86.37 \pm 13.66$ & 0.0993 & 0.1357 & 82.25 & 82.54 \\
\hline cmc & $52.41 \pm 3.70$ & $54.24 \pm 3.25$ & 0.3493 & 0.3517 & 47.08 & 47.19 \\
\hline derm & $97.86 \pm 2.45$ & $96.75 \pm 3.84$ & 0.1872 & 0.1776 & 86.27 & 87.59 \\
\hline german & $75.40 \pm 3.17$ & $77.80 \pm 2.94$ & 0.2713 & 0.2986 & 69.37 & 69.15 \\
\hline glass & $71.01 \pm 11.00$ & $76.64 \pm 10.61$ & 0.2951 & 0.3232 & 60.98 & 62.30 \\
\hline heart & $82.59 \pm 4.95$ & $84.44 \pm 4.88$ & 0.2407 & 0.2562 & 75.07 & 75.81 \\
\hline horse & $91.04 \pm 4.76$ & $93.49 \pm 3.83$ & 0.1465 & 0.1585 & 86.89 & 88.12 \\
\hline ICU & $92.08 \pm 2.31$ & $93.56 \pm 4.80$ & 0.0295 & 0.0317 & 87.06 & 89.58 \\
\hline iono & $95.73 \pm 4.51$ & $93.47 \pm 4.76$ & 0.2112 & 0.2330 & 86.73 & 87.61 \\
\hline iris & $95.33 \pm 6.32$ & $98.67 \pm 2.81$ & 0.0164 & 0.0180 & 94.49 & 98.57 \\
\hline movement & $80.44 \pm 16.59$ & $82.44 \pm 16.29$ & 0.3307 & 0.3255 & 59.48 & 61.37 \\
\hline pima & $76.17 \pm 4.57$ & $78.78 \pm 3.76$ & 0.2236 & 0.2332 & 72.52 & 73.27 \\
\hline rice & $83.89 \pm 8.78$ & $89.82 \pm 13.17$ & 0.1208 & 0.1212 & 79.97 & 83.37 \\
\hline spectf & $81.00 \pm 6.09$ & $82.48 \pm 7.91$ & 0.2420 & 0.2362 & 75.50 & 77.07 \\
\hline thyroid & $95.78 \pm 4.16$ & $96.26 \pm 4.86$ & 0.1083 & 0.1451 & 92.55 & 95.58 \\
\hline wiscon & $97.28 \pm 2.65$ & $97.86 \pm 2.36$ & 0.0366 & 0.0520 & 95.77 & 95.61 \\
\hline wdbc & $97.55 \pm 2.05$ & $97.72 \pm 1.66$ & 0.0838 & 0.0789 & 93.40 & 94.13 \\
\hline yeast & $71.62 \pm 4.32$ & $73.25 \pm 3.47$ & 0.1195 & 0.1383 & 70.55 & 70.86 \\
\hline zoo & $94.39 \pm 8.39$ & $94.39 \pm 8.39$ & 0.1045 & 0.0667 & 87.29 & 93.89 \\
\hline \hline Average & 85.06 & 86.83 & 0.1664 & 0.1757 & 79.35 & 80.89 \\
\hline
\end{tabular}

mean of the noise is zero, while we vary the standard deviation from 0 to 0.5 , and show the results in Fig. 4. As we can observe from there, DRMF is more robust with respect to attribute noise than $\mathrm{J} 48$, and performs similarly compared to Rotation Forest.

\section{Simulation and Experimental Analysis}

In this section, we compare DRMF with some other representative classification algorithms including J48, Rotation Forest, AdaBoostM1, and Bagging, on the 20 datasets from Table 1 . For all ensembles, base classifiers are generated using J48. For DRMF and Rotation Forest, we set $K=2$, the rate of bootstrap sampling to 0.75 and the number $L$ of base classifiers to 100 . The parameters of Bagging and AdaBoostM1 were kept as their default values in WEKA, while the number of base classifiers was also set to 100 . We performed standard 10-fold cross validation to compute the classification performance. Table 6 gives the classification accuracies on all datasets together with the standard deviations for all evaluated algorithms.

We further employ a test for statistical significance, namely the Nemenyi test [37], to compare the algorithms. In this test, the critical difference [16] for 

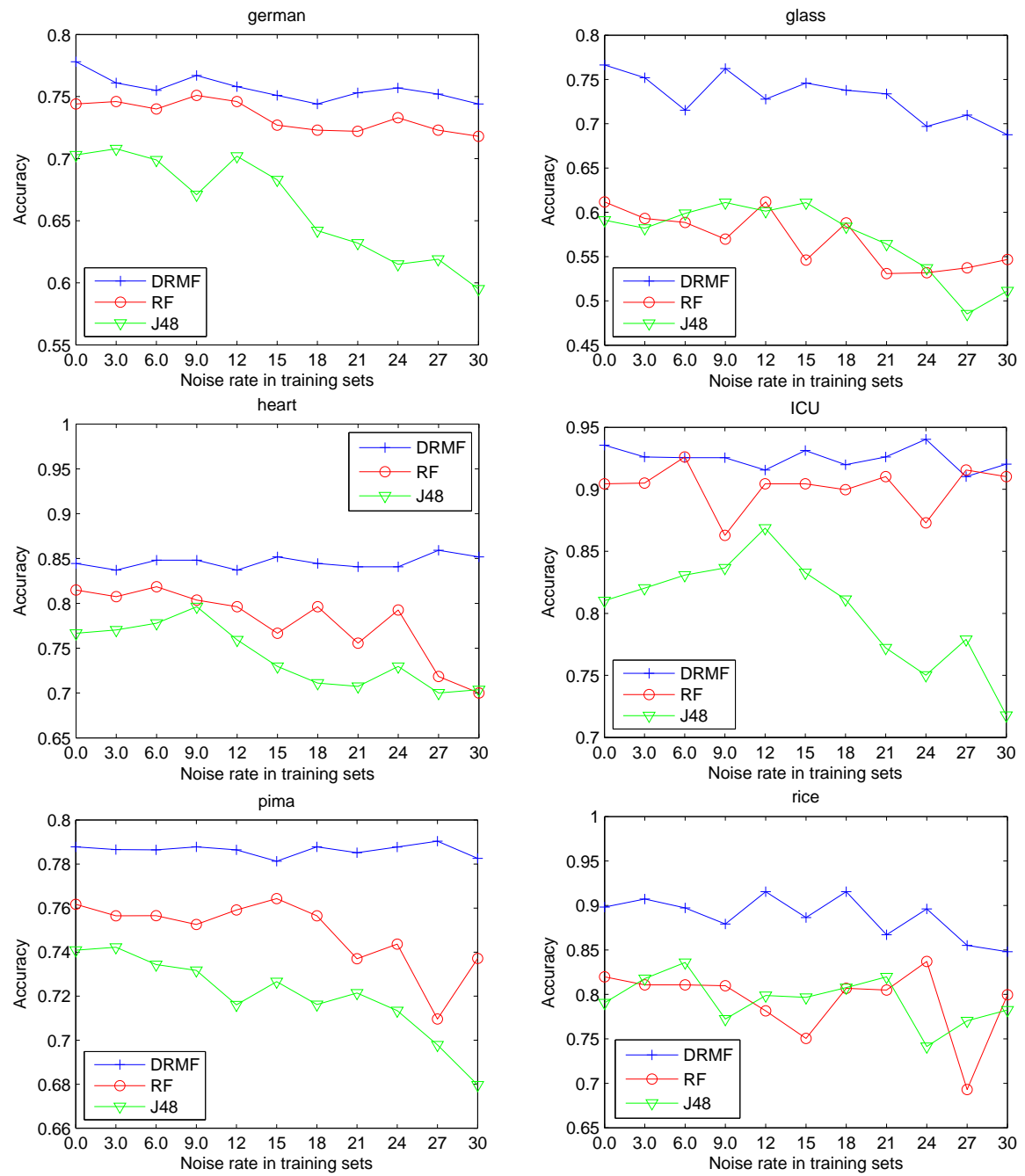

Figure 3: Variation of classification accuracies when varying the rate of class noise.

the five algorithms and 20 data sets at significance level $\alpha=0.05$ is

$$
C D=q_{0.05} \sqrt{\frac{k(k+1)}{6 \mathbb{N}}}=2.728 \times \sqrt{\frac{5 \times(5+1)}{6 \times 20}}=1.364,
$$

where $q_{0.05}$ is the critical value for the two-tailed Nemenyi test, $k$ is the number of the algorithms and $\mathbb{N}$ is the number of data sets.

The average ranks for DRMF, J48, Bagging, AdaBoostM1 and Rotation Forest were thus found to be $1.55,4.36,3.00,3.05$, and 3.03, respectively, and the average rank differences between DRMF and the other methods were 4.375$1.55=2.825>1.364,3.00-1.55=1.45>1.364,3.05-1.55=1.50>1.364$, 

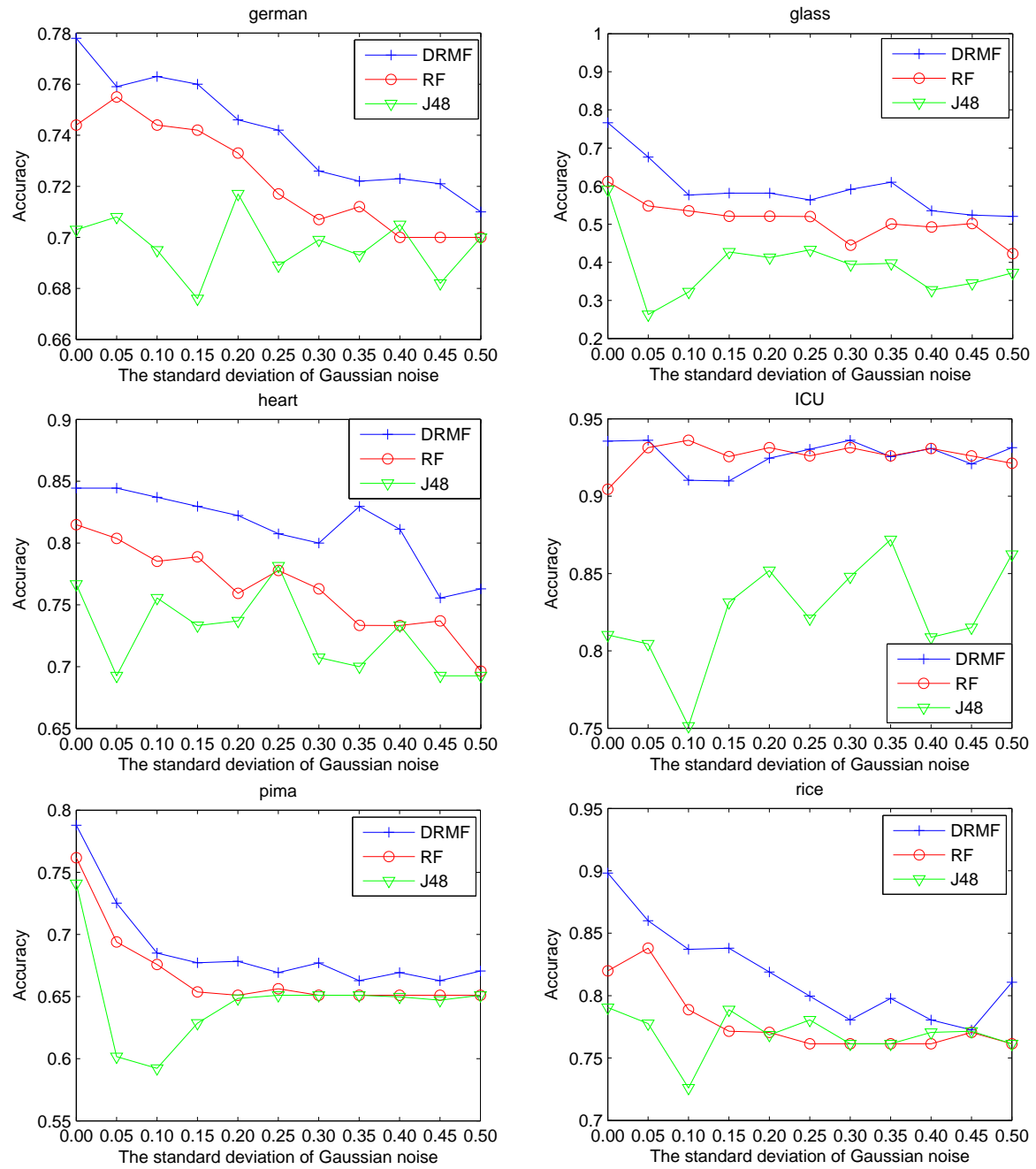

Figure 4: Variation of classification accuracies when varying the rate of attribute noise.

and $3.025-1.55=1.475>1.364$. Consequently, DRMF was shown to perform statistically significantly better than all other methods.

Next, we discuss why, compared with Rotation Forest, DRMF is able to further boost the classification performance. First, it can be seen that the parameter $K$ in DRMF and Rotation Forest was set to 2 in the above experiments. From Table 2 we know, that $K=2$ is suitable for DRMF. In order to verify whether $K=2$ is also suitable for Rotation Forest, the classification performances of Rotation Forest with different numbers of splits are given in Table 7 . From there, we can see that Rotation Forest also produces good performance if $K=2$. 
Table 6: Performance comparison with other classifiers.

\begin{tabular}{|c|c|c|c|c|c|}
\hline Data set & DRMF & J48 & Bagging & AdaBoostM1 & Rotation Forest \\
\hline \hline australian & $\mathbf{8 8 . 1 1} \pm \mathbf{3 . 4 8}$ & $83.05 \pm 5.40$ & $86.55 \pm 6.07$ & $86.10 \pm 5.01$ & $85.83 \pm 5.09$ \\
\hline crx & $\mathbf{8 6 . 3 7} \pm \mathbf{1 3 . 6 6}$ & $82.74 \pm 13.38$ & $83.91 \pm 15.48$ & $85.21 \pm 12.94$ & $83.04 \pm 17.89$ \\
\hline cmc & $54.24 \pm 3.25$ & $54.65 \pm 2.65$ & $53.84 \pm 3.32$ & $50.24 \pm 2.49$ & $\mathbf{5 4 . 7 2} \pm \mathbf{2 . 6 2}$ \\
\hline derm & $96.75 \pm 3.84$ & $93.73 \pm 4.50$ & $96.39 \pm 3.72$ & $95.12 \pm 3.30$ & $\mathbf{9 7 . 1 0} \pm \mathbf{3 . 1 9}$ \\
\hline german & $\mathbf{7 7 . 8 0} \pm \mathbf{2 . 9 4}$ & $70.30 \pm 3.40$ & $75.60 \pm 3.17$ & $76.00 \pm 3.53$ & $74.40 \pm 4.79$ \\
\hline glass & $\mathbf{7 6 . 6 4} \pm \mathbf{1 0 . 6 1}$ & $59.11 \pm 13.53$ & $68.17 \pm 11.17$ & $72.89 \pm 17.04$ & $61.17 \pm 11.68$ \\
\hline heart & $\mathbf{8 4 . 4 4} \pm \mathbf{4 . 8 8}$ & $76.67 \pm 5.25$ & $82.22 \pm 7.77$ & $80.00 \pm 5.84$ & $81.48 \pm 6.98$ \\
\hline horse & $93.49 \pm 3.83$ & $96.19 \pm 2.65$ & $97.27 \pm 2.27$ & $\mathbf{9 7 . 5 6} \pm \mathbf{0 . 8 6}$ & $91.02 \pm 4.64$ \\
\hline ICU & $\mathbf{9 3 . 5 6} \pm \mathbf{4 . 8 0}$ & $81.03 \pm 29.12$ & $84.14 \pm 29.72$ & $84.14 \pm 29.83$ & $90.45 \pm 12.03$ \\
\hline iono & $93.47 \pm 4.76$ & $89.24 \pm 7.90$ & $91.21 \pm 5.83$ & $93.22 \pm 4.62$ & $\mathbf{9 4 . 3 4} \pm \mathbf{4 . 9 4}$ \\
\hline iris & $\mathbf{9 8 . 6 7} \pm \mathbf{2 . 8 1}$ & $96.00 \pm 3.44$ & $94.67 \pm 6.13$ & $96.00 \pm 3.44$ & $95.33 \pm 3.22$ \\
\hline movement & $\mathbf{8 2 . 4 4} \pm \mathbf{1 6 . 2 9}$ & $62.44 \pm 16.89$ & $68.89 \pm 17.73$ & $74.11 \pm 18.82$ & $82.00 \pm 20.92$ \\
\hline pima & $\mathbf{7 8 . 7 8} \pm \mathbf{3 . 7 6}$ & $74.09 \pm 5.87$ & $76.43 \pm 4.89$ & $73.57 \pm 3.74$ & $76.17 \pm 3.39$ \\
\hline rice & $\mathbf{8 9 . 8 2} \pm \mathbf{1 3 . 1 7}$ & $79.05 \pm 10.04$ & $83.89 \pm 8.78$ & $83.89 \pm 8.78$ & $81.98 \pm 8.95$ \\
\hline spectf & $\mathbf{8 2 . 4 8} \pm \mathbf{7 . 9 1}$ & $73.47 \pm 8.47$ & $78.78 \pm 10.74$ & $78.46 \pm 9.85$ & $80.26 \pm 6.15$ \\
\hline thyroid & $\mathbf{9 6 . 2 6} \pm \mathbf{4 . 8 6}$ & $93.48 \pm 5.93$ & $94.87 \pm 6.43$ & $94.00 \pm 10.12$ & $95.78 \pm 7.25$ \\
\hline wiscon & $\mathbf{9 7 . 8 6} \pm \mathbf{2 . 3 6}$ & $94.57 \pm 2.10$ & $96.43 \pm 3.03$ & $95.71 \pm 3.01$ & $96.57 \pm 2.87$ \\
\hline wdbc & $\mathbf{9 7 . 7 2} \pm \mathbf{1 . 6 6}$ & $92.98 \pm 3.96$ & $96.31 \pm 3.36$ & $97.19 \pm 1.90$ & $97.19 \pm 2.22$ \\
\hline yeast & $73.25 \pm 3.47$ & $74.53 \pm 4.44$ & $\mathbf{7 6 . 6 8} \pm \mathbf{5 . 5 5}$ & $73.52 \pm 3.59$ & $71.89 \pm 3.88$ \\
\hline zoo & $94.39 \pm 8.39$ & $90.76 \pm 10.26$ & $93.30 \pm 7.07$ & $\mathbf{9 6 . 3 8} \pm \mathbf{5 . 7 5}$ & $90.65 \pm 9.13$ \\
\hline
\end{tabular}

Table 7: Classification performances of RF with different numbers of splits.

\begin{tabular}{|c|c|c|c|c|}
\hline Data set & $\mathrm{K}=2$ & $\mathrm{~K}=3$ & $\mathrm{~K}=4$ & $\mathrm{~K}=5$ \\
\hline \hline australian & $85.83 \pm 5.09$ & $85.80 \pm 3.83$ & $\mathbf{8 6 . 0 9} \pm \mathbf{3 . 5 3}$ & $84.34 \pm 3.62$ \\
\hline crx & $83.04 \pm 17.89$ & $82.18 \pm 17.54$ & $82.89 \pm 15.68$ & $\mathbf{8 3 . 7 5} \pm \mathbf{1 6 . 0 0}$ \\
\hline cmc & $\mathbf{5 4 . 7 2} \pm \mathbf{2 . 6 2}$ & $52.68 \pm 2.60$ & $53.02 \pm 4.37$ & $52.89 \pm 2.65$ \\
\hline derm & $97.10 \pm 3.19$ & $\mathbf{9 7 . 7 8} \pm \mathbf{2 . 8 7}$ & $97.26 \pm 2.93$ & $97.02 \pm 2.99$ \\
\hline german & $74.40 \pm 4.79$ & $\mathbf{7 5 . 3 0} \pm \mathbf{3 . 9 7}$ & $75.10 \pm 5.09$ & $74.80 \pm 4.87$ \\
\hline glass & $61.17 \pm 11.68$ & $66.33 \pm 10.64$ & $\mathbf{7 1 . 4 2} \pm \mathbf{1 3 . 5 3}$ & $60.22 \pm 15.34$ \\
\hline heart & $81.48 \pm 6.98$ & $\mathbf{8 4 . 8 1} \pm \mathbf{4 . 0 8}$ & $83.33 \pm 6.11$ & $77.41 \pm 9.31$ \\
\hline horse & $91.02 \pm 4.64$ & $91.84 \pm 3.66$ & $91.56 \pm 3.77$ & $\mathbf{9 2 . 6 6} \pm \mathbf{3 . 3 9}$ \\
\hline ICU & $\mathbf{9 0 . 4 5} \pm \mathbf{1 2 . 0 3}$ & $\mathbf{9 0 . 4 5} \pm \mathbf{1 3 . 9 2}$ & $87.29 \pm 17.86$ & $87.29 \pm 21.75$ \\
\hline iono & $\mathbf{9 4 . 3 4} \pm \mathbf{4 . 9 4}$ & $93.50 \pm 4.95$ & $94.06 \pm 5.21$ & $93.50 \pm 4.95$ \\
\hline iris & $95.33 \pm 3.22$ & $94.00 \pm 4.92$ & $\mathbf{9 6 . 0 0} \pm \mathbf{3 . 4 4}$ & $\mathbf{9 6 . 0 0} \pm \mathbf{3 . 4 4}$ \\
\hline movement & $\mathbf{8 2 . 0 0} \pm \mathbf{2 0 . 9 2}$ & $80.89 \pm 21.25$ & $78.11 \pm 23.44$ & $80.33 \pm 20.03$ \\
\hline pima & $\mathbf{7 6 . 1 7} \pm \mathbf{3 . 3 9}$ & $75.13 \pm 5.20$ & $74.74 \pm 5.04$ & $73.57 \pm 3.65$ \\
\hline rice & $\mathbf{8 1 . 9 8} \pm \mathbf{8 . 9 5}$ & $79.05 \pm 10.04$ & $79.96 \pm 10.70$ & $79.05 \pm 10.04$ \\
\hline spectf & $80.26 \pm 6.15$ & $\mathbf{8 0 . 9 9} \pm \mathbf{7 . 4 6}$ & $78.86 \pm 7.67$ & $81.01 \pm 5.52$ \\
\hline thyroid & $\mathbf{9 5 . 7 8} \pm \mathbf{7 . 2 5}$ & $93.48 \pm 7.92$ & $93.96 \pm 8.17$ & $93.48 \pm 5.93$ \\
\hline wiscon & $96.57 \pm 2.87$ & $\mathbf{9 7 . 4 3} \pm \mathbf{2 . 9 2}$ & $96.00 \pm 3.29$ & $92.99 \pm 3.71$ \\
\hline wdbc & $97.19 \pm 2.22$ & $97.38 \pm 2.64$ & $\mathbf{9 7 . 7 3} \pm \mathbf{2 . 3 3}$ & $97.02 \pm 2.62$ \\
\hline yeast & $\mathbf{7 1 . 8 9} \pm \mathbf{3 . 8 8}$ & $70.55 \pm 5.80$ & $70.68 \pm 5.80$ & $70.68 \pm 6.05$ \\
\hline zoo & $90.65 \pm 9.13$ & $90.28 \pm 8.34$ & $88.65 \pm 9.04$ & $\mathbf{9 2 . 3 9} \pm \mathbf{9 . 2 4}$ \\
\hline \hline Average & 84.07 & 83.99 & 83.84 & 83.02 \\
\hline
\end{tabular}


Table 8: Classification performances with different rotation and fusion strategies.

\begin{tabular}{|c|c|c|c|c|}
\hline Data set & RF & DRSF & PRMF & DRMF \\
\hline \hline australian & $85.83 \pm 5.09$ & $86.09 \pm 3.88$ & $88.02 \pm 3.95$ & $\mathbf{8 8 . 1 1} \pm \mathbf{3 . 4 8}$ \\
\hline crx & $83.04 \pm 17.89$ & $83.61 \pm 18.39$ & $85.94 \pm 14.06$ & $\mathbf{8 6 . 3 7} \pm \mathbf{1 3 . 6 6}$ \\
\hline cmc & $54.72 \pm 2.62$ & $52.41 \pm 3.70$ & $\mathbf{5 5 . 6 7} \pm \mathbf{2 . 1 5}$ & $54.24 \pm 3.25$ \\
\hline derm & $97.10 \pm 3.19$ & $\mathbf{9 7 . 8 6} \pm \mathbf{2 . 4 5}$ & $97.26 \pm 2.62$ & $96.75 \pm 3.84$ \\
\hline german & $74.40 \pm 4.79$ & $75.40 \pm 3.17$ & $76.50 \pm 5.10$ & $\mathbf{7 7 . 8 0} \pm \mathbf{2 . 9 4}$ \\
\hline glass & $61.17 \pm 11.68$ & $58.81 \pm 11.78$ & $75.28 \pm 8.38$ & $\mathbf{7 6 . 6 4} \pm \mathbf{1 0 . 6 1}$ \\
\hline heart & $81.48 \pm 6.98$ & $82.59 \pm 4.95$ & $83.70 \pm 5.58$ & $\mathbf{8 4 . 4 4} \pm \mathbf{4 . 8 8}$ \\
\hline horse & $91.02 \pm 4.64$ & $91.04 \pm 4.76$ & $92.67 \pm 4.42$ & $\mathbf{9 3 . 4 9} \pm \mathbf{3 . 8 3}$ \\
\hline ICU & $90.45 \pm 12.03$ & $91.55 \pm 3.34$ & $91.98 \pm 11.08$ & $\mathbf{9 3 . 5 6} \pm \mathbf{4 . 8 0}$ \\
\hline iono & $94.34 \pm 4.94$ & $\mathbf{9 5 . 7 3} \pm \mathbf{4 . 5 1}$ & $95.16 \pm 2.74$ & $93.47 \pm 4.76$ \\
\hline iris & $95.33 \pm 3.22$ & $95.33 \pm 6.32$ & $96.00 \pm 3.44$ & $\mathbf{9 8 . 6 7} \pm \mathbf{2 . 8 1}$ \\
\hline movement & $82.00 \pm 20.92$ & $80.44 \pm 16.59$ & $\mathbf{8 2 . 8 9} \pm \mathbf{1 8 . 1 3}$ & $82.44 \pm 16.29$ \\
\hline pima & $76.17 \pm 3.39$ & $76.17 \pm 4.57$ & $76.05 \pm 3.99$ & $\mathbf{7 8 . 7 8} \pm \mathbf{3 . 7 6}$ \\
\hline rice & $81.98 \pm 8.95$ & $83.89 \pm 8.78$ & $87.82 \pm 10.99$ & $\mathbf{8 9 . 8 2} \pm \mathbf{1 3 . 1 7}$ \\
\hline spectf & $80.26 \pm 6.15$ & $81.00 \pm 6.09$ & $82.39 \pm 5.96$ & $\mathbf{8 2 . 4 8} \pm \mathbf{7 . 9 1}$ \\
\hline thyroid & $95.78 \pm 7.25$ & $95.78 \pm 4.16$ & $95.76 \pm 6.12$ & $\mathbf{9 6 . 2 6} \pm \mathbf{4 . 8 6}$ \\
\hline wiscon & $96.57 \pm 2.87$ & $97.28 \pm 2.65$ & $97.28 \pm 2.47$ & $\mathbf{9 7 . 8 6} \pm \mathbf{2 . 3 6}$ \\
\hline wdbc & $97.19 \pm 2.22$ & $97.55 \pm 2.05$ & $97.18 \pm 3.04$ & $\mathbf{9 7 . 7 2} \pm \mathbf{1 . 6 6}$ \\
\hline yeast & $71.89 \pm 3.88$ & $71.62 \pm 4.32$ & $73.11 \pm 3.18$ & $\mathbf{7 3 . 2 5} \pm \mathbf{3 . 4 7}$ \\
\hline zoo & $90.65 \pm 9.13$ & $93.76 \pm 10.06$ & $92.28 \pm 8.00$ & $\mathbf{9 4 . 3 9} \pm \mathbf{8 . 3 9}$ \\
\hline \hline Average & 84.07 & 84.40 & 86.15 & 86.83 \\
\hline
\end{tabular}

The difference between DRMF and Rotation Forest mainly comprises two parts: the use of Double Rotation to generate the base classifiers, and Margin Based Pruning. Thus, we explore whether they are both necessary for improving the classification performance of the ensemble. For this, we test four combinations: Rotation Forest (RF); a combination of Double Rotation and the fusion strategy in Rotation Forest i.e. simple voting of all base classifiers (DRSF), a combination of PCA rotation and Margin Based Pruning (PRMF), and DRMF. The results are presented in Table 8. As we can confirm from there, both Double Rotation and Margin Based Pruning are indeed useful for improving the classification performance.

Further, we compute the margin distribution of the ensembles generated using the above four classification algorithms, where the margin of a sample is computed as the difference between the number of correct votes and the maximum number of the votes received by any wrong label [42]. A large margin is understood as a "confident" classification, and thus we would desire that large margins of the training samples are derived. Fig. 5 shows the margin distribution when RF, DRSF, PRMF and DRMF are used. We can observe, that compared with RF, DRMF improves the margin distribution on the training set, which confirms that both Double Rotation and Margin Based Pruning are helpful for improving the margin distribution.

So, why does Double Rotation improve the margin distribution of the training set? From Theorem 1, we know that the margin of the training samples has 

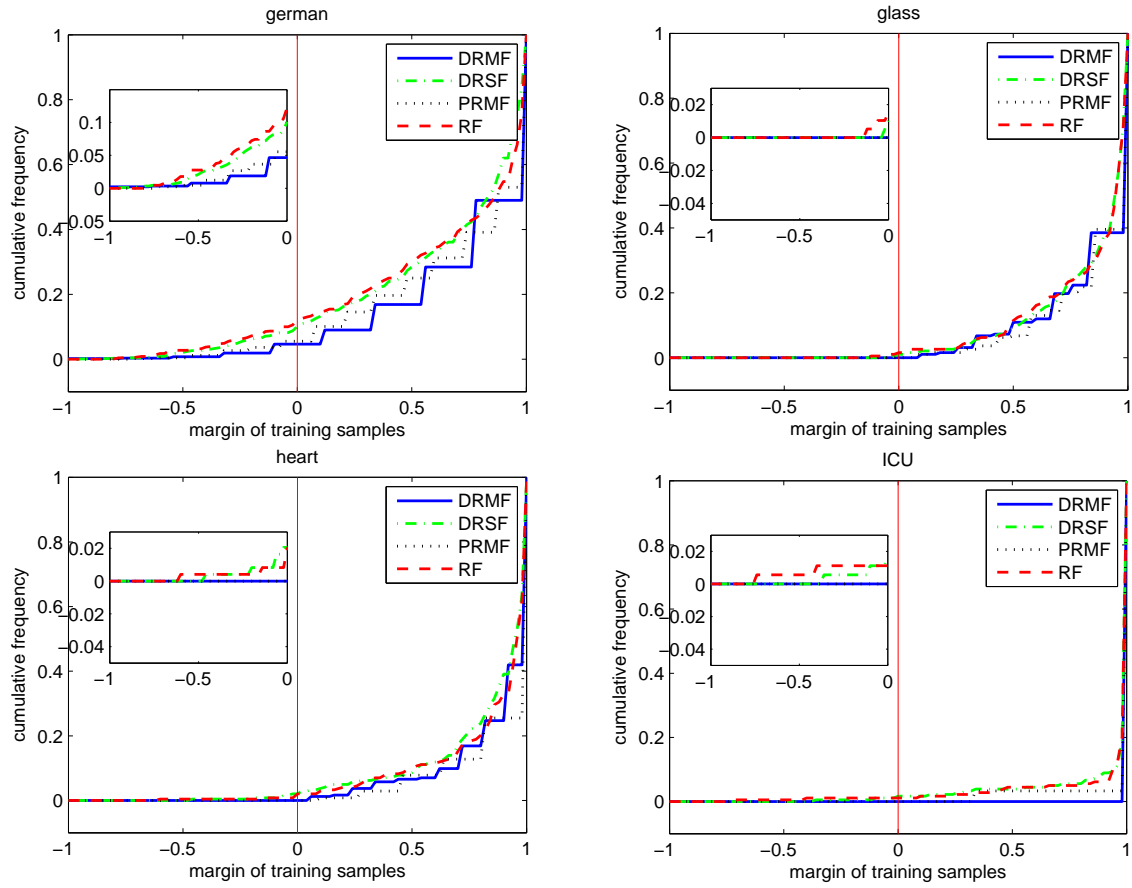

ICU
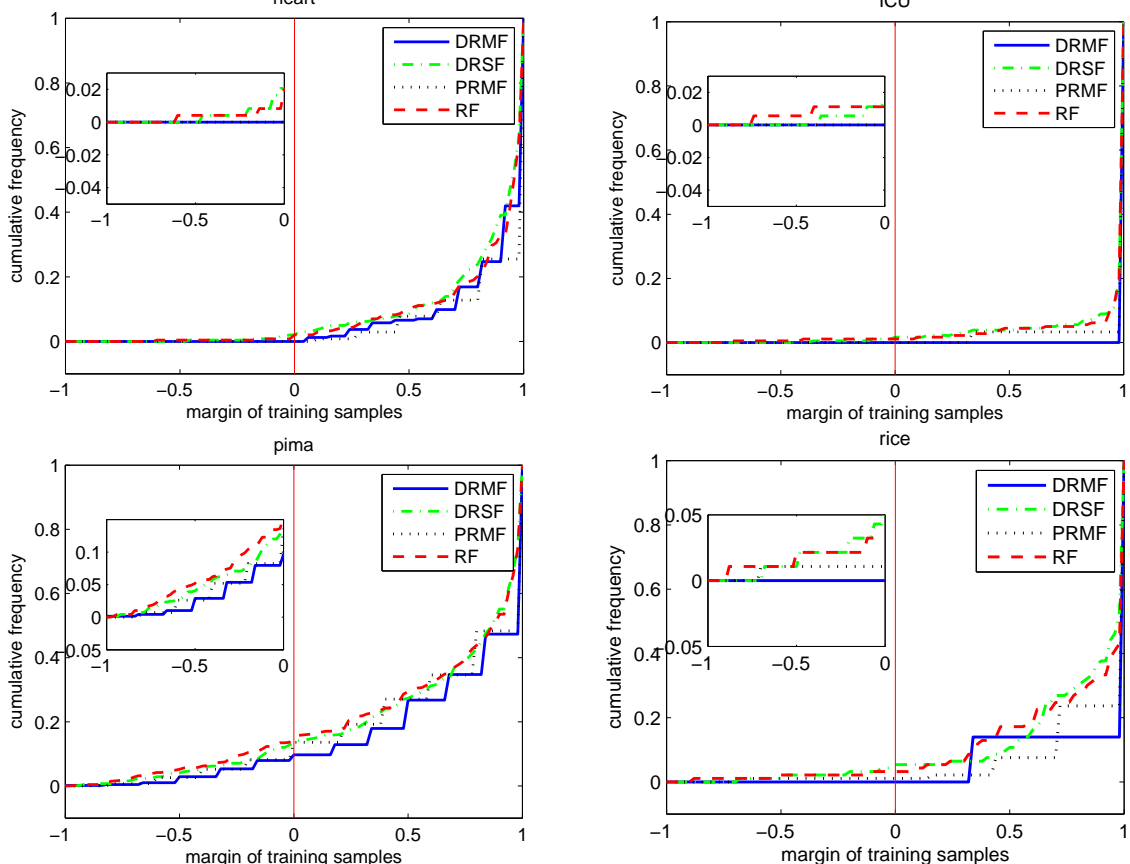

Figure 5: Margin cumulative frequency of training samples for RF, DRSF, PRMF and DRMF.

an underlying relationship with the diversity of the base classifiers. We thus compare the diversity of the base classifiers when employing Double Rotation and PCA rotation, and show the results in Fig. 6. From there we can notice that the diversity among base classifiers is consistently enhanced after Double Rotation. However, from Fig. 7 we see that the average accuracies of the two kinds of base classifiers are almost the same. Consequently, we can derive that the improvements of the margin distribution come from the diversity, and not from the improved accuracies of the base classifiers.

Finally, we conducted some experiments to validate the effectiveness of the proposed Margin Based Pruning (Margin-P) algorithm and compared it with 


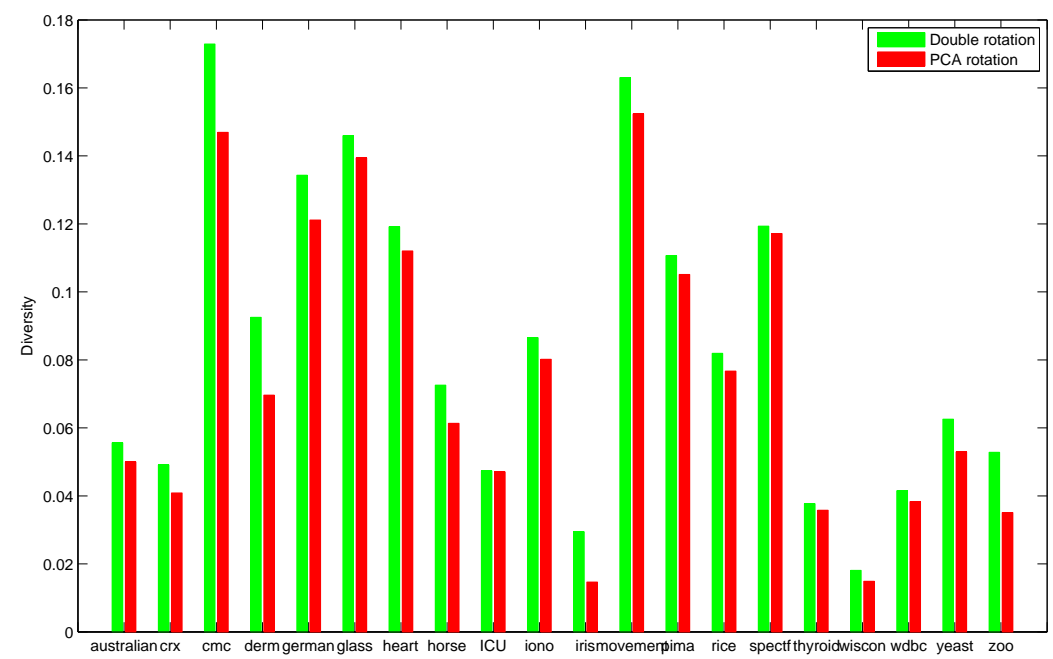

Figure 6: Diversity of Double Rotation and PCA Rotation.

other ensemble pruning techniques based on the margin including MeanD-M [52] and the improved version of MDM $[32,33]$. MeanD-M optimizes the average margin via a backward elimination strategy. In particular, it ranks the contribution and importance of every base classifier $\mathcal{C}_{j}$ in the temporary ensemble $\Gamma$ by observing the decrease of the average margin when removing $\mathcal{C}_{j}$ from the ensemble. During each step, the least important classifier $\mathcal{C}_{\text {min }}$ with the minimum decrease of the average margin is eliminated from the ensemble and the ensemble thus shrinks to its subset $\Gamma^{\prime}=\Gamma \backslash \mathcal{C}_{\text {min }}$. Then, the base classifiers in $\Gamma^{\prime}$ are reordered and the above process is repeated. MDM selects base classifiers via a forward selection strategy where base classifiers are sequentially added based on a specified rule. In particular, the classifier selected in the $u$-th iteration is

$$
s_{u}=\arg \underset{j}{\min } \mathrm{d}\left(\mathbf{o}, \frac{1}{u}\left(\mathbf{c}_{j}+\sum_{t=1}^{u-1} \mathbf{c}_{s_{t}}\right)\right),
$$

where $\mathbf{c}_{j}$ is the $N$-dimensional signature vector of $\mathcal{C}_{j}$ whose $i$-th component $\left(\mathbf{c}_{j}\right)_{i}$ is 1 if the sample $x_{i}$ is correctly classified by $\mathcal{C}_{j}$ and is -1 otherwise. $j$ runs through the classifiers outside the temporary ensemble and $d\left(\mathbf{v}_{1}, \mathbf{v}_{2}\right)$ is the distance between vectors $\mathbf{v}_{1}$ and $\mathbf{v}_{2}$. In [33], the objective point $\mathbf{o}$ is placed in the first quadrant with equal components $\mathbf{o}_{i}=p$ (e.g., $p=0.075$ ). An improved version of MDM is proposed [32], which uses a moving objective point o that allows $p(u)$ to vary with the size of the sub-ensemble $u$. Exploratory experiments show that a value $p(u) \propto \sqrt{u}$ is appropriate. improved version for comparison with our method. 

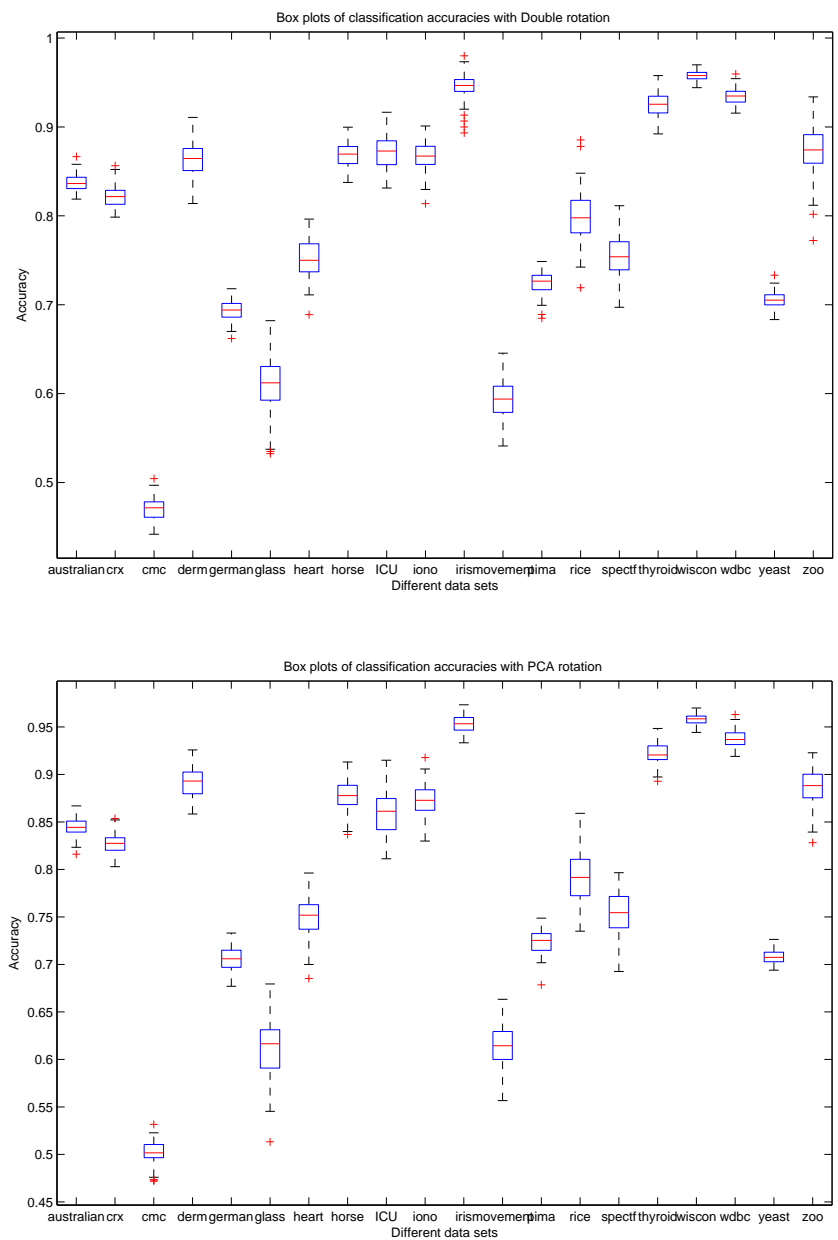

Figure 7: Box plots of classification accuracies with different rotation strategies.

In MeanD-M, base classifiers are eliminated from the original ensemble one by one and the sub-ensemble with the best accuracy on the test set is used to estimate its performance. Thus, we also use the best accuracy on the test set to estimate the classification performance for MDM and Margin-P. The results of the experiment are given in Table 9. From there, we can confirm that our algorithm does indeed performs better than the pruning strategies in most cases.

\section{Conclusions and Future Work}

Ensemble learning is an effective approach to improve the generalization performance of a classification system. In this paper, we have proposed Double 
Table 9: The best test performances based on different pruning methods.

\begin{tabular}{|c|c|c|c|}
\hline Data set & MeanD-M & MDM & Margin-P \\
\hline \hline australian & $88.41 \pm 3.48$ & $87.54 \pm 2.99$ & $\mathbf{8 8 . 9 8} \pm \mathbf{3 . 1 6}$ \\
\hline crx & $86.22 \pm 15.11$ & $87.23 \pm 14.20$ & $\mathbf{8 7 . 8 1} \pm \mathbf{1 3 . 7 1}$ \\
\hline cmc & $55.94 \pm 2.97$ & $56.08 \pm 4.00$ & $\mathbf{5 6 . 6 2} \pm \mathbf{3 . 4 8}$ \\
\hline derm & $\mathbf{9 8 . 4 1} \pm \mathbf{2 . 2 1}$ & $98.13 \pm 2.16$ & $\mathbf{9 8 . 4 1} \pm \mathbf{2 . 2 1}$ \\
\hline german & $78.60 \pm 2.17$ & $79.00 \pm 2.49$ & $\mathbf{7 9 . 5 0} \pm \mathbf{3 . 0 6}$ \\
\hline glass & $77.60 \pm 10.62$ & $75.24 \pm 7.89$ & $\mathbf{7 9 . 9 4} \pm \mathbf{9 . 8 9}$ \\
\hline heart & $86.30 \pm 3.92$ & $85.93 \pm 4.55$ & $\mathbf{8 7 . 0 4} \pm \mathbf{3 . 1 5}$ \\
\hline horse & $94.59 \pm 4.37$ & $93.50 \pm 3.64$ & $\mathbf{9 4 . 8 5} \pm \mathbf{3 . 7 0}$ \\
\hline ICU & $93.61 \pm 5.31$ & $94.56 \pm 5.06$ & $\mathbf{9 4 . 6 1} \pm \mathbf{4 . 2 9}$ \\
\hline iono & $\mathbf{9 7 . 1 2} \pm \mathbf{3 . 6 6}$ & $96.60 \pm 3.71$ & $96.84 \pm 3.27$ \\
\hline iris & $98.00 \pm 4.50$ & $97.33 \pm 3.44$ & $\mathbf{9 8 . 6 7} \pm \mathbf{2 . 8 1}$ \\
\hline movement & $85.33 \pm 16.04$ & $83.67 \pm 16.06$ & $\mathbf{8 5 . 4 4} \pm \mathbf{1 5 . 8 7}$ \\
\hline pima & $78.91 \pm 3.15$ & $79.43 \pm 4.10$ & $\mathbf{8 0 . 8 6} \pm \mathbf{3 . 3 9}$ \\
\hline rice & $91.55 \pm 8.24$ & $91.34 \pm 8.29$ & $\mathbf{9 4 . 3 6} \pm \mathbf{6 . 4 8}$ \\
\hline spectf & $85.86 \pm 4.25$ & $85.11 \pm 7.27$ & $\mathbf{8 6 . 6 2} \pm \mathbf{5 . 5 6}$ \\
\hline thyroid & $97.19 \pm 4.58$ & $96.69 \pm 4.51$ & $\mathbf{9 7 . 6 4} \pm \mathbf{4 . 6 2}$ \\
\hline wiscon & $98.00 \pm 2.25$ & $\mathbf{9 8 . 4 3} \pm \mathbf{1 . 9 6}$ & $98.14 \pm 2.13$ \\
\hline wdbc & $\mathbf{9 8 . 6 0} \pm \mathbf{1 . 3 8}$ & $98.25 \pm 1.42$ & $98.42 \pm 1.29$ \\
\hline yeast & $73.92 \pm 3.41$ & $74.12 \pm 4.08$ & $\mathbf{7 4 . 4 6} \pm \mathbf{2 . 3 2}$ \\
\hline zoo & $\mathbf{9 5 . 3 9} \pm \mathbf{8 . 4 1}$ & $\mathbf{9 5 . 3 9} \pm \mathbf{8 . 4 1}$ & $\mathbf{9 5 . 3 9} \pm \mathbf{8 . 4 1}$ \\
\hline \hline Average & 87.98 & 87.68 & 88.73 \\
\hline
\end{tabular}

Rotation Margin Forest (DRMF) as an effective new ensemble learning algorithm. The idea of DRMF is to improve the generalization performance by improving the margin distribution on the training set. Extensive experimental results on 20 benchmark datasets confirm that DRMF provides a competent ensemble learner, and allows us to draw several conclusions: (1) Double Rotation with PCA and LSDA is able to generate diverse base classifiers; (2) The margin distribution of the ensemble system is improved if a set of diverse base classifiers is exploited by optimizing a regularized loss function, and consequently the classification performance of the ensemble is enhanced; (3) The DRMF algorithm outperforms classical ensemble learning techniques such as Bagging, AdaBoostM1 and Rotation Forest.

Our work is motivated by the idea that diversity among base classifiers can improve the margin distribution of the ensemble. However, no deep discussion on this issue has been reported so far. Further theoretical analysis on the relationship between diversity and margin is thus required. While in this paper, we use Double Rotation to create diversity, some other techniques could also be introduced. A systematic discussion on generating diversity would therefore present an important task. Although DRMF is presented as an approach to create homogenous ensembles (e.g., based only on decision trees as the base classifiers), it is straightforward to use DRMF to learn and prune heterogeneous classifiers for ensemble learning. Exploring the effectiveness of DRMF in such a setting might be an interesting avenue. 


\section{Acknowledgments}

This work is supported by the National Program on Key Basic Research Project under Grant 2013CB329304, National Natural Science Foundation of China under Grants 61222210, 60873140, 61170107, 61073125, 61071179, 60963006, and 11078010, National Science Fund for Distinguished Young Scholars under Grant 50925625 and the Program for New Century Excellent Talents in University (No. NCET-12-0399, NCET-08-0155, and NCET-08-0156).

\section{References}

[1] M. Aksela and J. Laaksonen, Using diversity of errors for selecting members of a committee classifier, Pattern Recognition, 39 (2006) 608-623.

[2] F. Aiolli and A. Sperduti, A re-weighting strategy for improving margins, Artificial Intelligence, 137 (2002) 197-216.

[3] C. Blake, E. Keogh and C. J. Merz (1998), UCI Repository of Machine Learning Databases. Dept. Inf. Comput. Sci., Univ. California, Irvine, CA. [Online]. Available: http://www.ics.uci.edu/ mlearn/MLRepository. html.

[4] L. Breiman, Bagging predictors, Machine Learning, 24 (1996) 123-140.

[5] L. Breiman, Stacked regressions, Machine Learning, 24 (1996) 49-64.

[6] P. L. Bartlett, For valid generalization, the size of the weights is more important than the size of the network, In Advances inNeural Information Processing Systems, vol. 9, 1997.

[7] R. Bryll, R. Gutierrez-Osuna and F. Quek, Attribute bagging: improving accuracy of classifier ensembles by using random feature subsets, Pattern Recognition, 36 (2003) 1291-1302.

[8] B. E. Boser, I. M. Guyon and V. N. Vapnik, A training algorithm for optimal margin classifiers, In Proceedings of the Fifth Annual ACM Workshop on Computational Learning Theory, pp. 144-152, 1992.

[9] J. A. Benediktsson, J. R. Sveinsson, O. K. Ersoy and P. H. Swain, Parallel consensual neural networks, IEEE Transactions on Neural Networks, 8 (1) (1997) 54-64.

[10] K. J. Cherkauer, Human expert level performance on a scientific image analysis task by a system using combined artificial neural networks, in: Proceedings of 13th AAAI workshop on integrating multiple learned models for improving and scaling machine algorithms, pp. 15-21, 1996.

[11] K. Crammer, R. Gilad-Bachrach, A. Navot and A. Tishby, Margin analysis of the LVQ algorithm, Advances in Neural Information Processing Systems, 15 (2003) 462-469. 
[12] D. Cai, X. F. He, K. Zhou, J. W. Han and H. J. Bao, Locality Sensitive Discriminant Analysis, International Joint Conference on Artificial Intelligence, pp. 708-713, 2007.

[13] H. Chen, P. Tino and X. Yao, Predictive ensemble pruning by expectation propagation, IEEE Transactions on Knowledge and Data Engineering, 21 (7) (2009) 999-1013.

[14] C. Cortes and V. Vapnik, Support-vector networks, Machine Learning, 20 (3) (1995) 273-297.

[15] Q. Dai, A competitive ensemble pruning approach based on cross-validation technique, Knowledge-Based Systems, 37 (2013) 394-414.

[16] J. Demšar, Statistical comparisons of classifiers over multiple data sets, Journal of Machine Learning Research, 7 (1) (2006) 1-30.

[17] Y. Freund, Boosting a weak learning algorithm by majority, Information and computation, 121 (1996) 256-285.

[18] S. Floyd and M. Marmuth, Sample compression learnability, and the VapnikC Chervonenkis dimension, Machine Learning, 21 (3) (1995) 269-304.

[19] G. Fumera and F. Roli, A theoretical and experiment alanalysis of linear combiners for multiple classifier systems, IEEE Transactions on Pattern Analysis and Machine Intelligence, 27 (6) (2005) 942-956.

[20] Y. Freund and R. E. Schapire, A decision-theoretic generalization of online learning and an application to boosting, J. Comput. Syst. Sci., 55 (1) (1997) 119-139.

[21] Y. Freund and R. E. Schapire, Large Margin Classification Using the Perceptron Algorithm, Machine Learning, 37 (3) (1999) 277-296.

[22] T. Graepel, R. Herbrich and J. Shawe-Taylor, Generalization error bounds for sparse linear classifiers, in: 13th Annual Conference on Computational Learning Theory, pp. 298-303, 2000.

[23] A. J. Grove and D. Schuurmans, Boosting in the limit: maximizing the margin of learned ensembles, in:Proceedings of the 15th National Conference on Artificial Intelligence,American Association for Artificial Intelligence, Menlo Park, CA, USA, pp. 692-699, 1998.

[24] D. Hernández-Lobato, G. Martínez-Muñoz and A. Suárez, Empirical analysis and evaluation of approximate techniques for pruning regression bagging ensembles, Neurocomputing, 74 (12-13) (2011) 2250-2264.

[25] Q. H. Hu, D. R. Yu and M. Y. Wang, Constructing Rough Decision Forests, D. Slezak et al. (Eds.), RSFDGrC 2005, Lecture Notes in Artificial Intelligence, 3642 (2005) 147-156. 
[26] Q. H. Hu, P. F. Zhu, Y. B. Yang and D. R. Yu, Large-margin nearest neighbor classifiers via sample weight learning, Neurocomputing, 74 (2011) 656-660.

[27] M. Hall, E. Frank, G. Holmes, B. Pfahringer, P. Reutemann and I. H. Witten, The WEKA Data Mining Software: An Update, SIGKDD Explorations, 11 (1) (2009) 10-18.

[28] J. Kittler, M. Hatef, R. P. W. Duin and J. Matas, On combining classifiers, IEEE Transactions on Pattern Analysis and Machine Intelligence, 20 (3) (1998) 226-239.

[29] L. I. Kuncheva, Switching Between Selection and Fusion in Combining Classifiers: An Experiment, IEEE Transactions on Systems, Man and $\mathrm{Cy}-$ bernetics, Part-B: Cybernetics, 32 (2) (2002) 146-156.

[30] L. Kuncheva and C. Whitaker, Measures of diversity in classifier ensembles and their relationship with the ensemble accuracy, Machine Learning, 51 (2003) 181-207.

[31] D. D. Margineantu and T. G. Dietterich, Pruning adaptive boosting, in: Proceedings of the 14th International Conference on Machine Learning, Morgan Kaufmann Publishers Inc.,San Francisco, CA, USA, pp. 211-218, 1997.

[32] G. Martínez-Muñoz, D. Hernandez-Lobato and A.Suarez, An analysis of ensemble pruning techniques based on ordered aggregation, IEEE Transactions on Pattern Analysis and Machine Intelligence, 31 (2) (2009) 245-259.

[33] G. Martínez-Muñoz and A. Suárez, Aggregation ordering in bagging, in:Proceedings of the International Conference on Artificial Intelligence and Applications, pp. 258-263, 2004.

[34] G. Martínez-Muñoz and A. Suárez, Pruning in ordered bagging ensembles, in: Proceedings of the 23th International Conference on Machine Learning, pp. 609-616, 2006.

[35] G. Martínez-Muñoz and A. Suárez, Using boosting to prune bagging ensembles, Pattern Recognition Letters, 28 (1) (2007) 156-165.

[36] R. Maclin and J. W. Shavlik, Combining the predictions of multiple classifiers: using competitive learning to initialize neural networks, in: Proceedings of the 14th international joint conference on artificial intelligence, Morgan Kaufmann, San Mateo, CA, pp. 524-530, 1995.

[37] P. B. Nemenyi. Distribution-free multiple comparisons, $\mathrm{PhD}$ thesis, Princeton University, 1963.

[38] A. Rahman and B. Verma, Ensemble classifier generation using nonuniform layered clustering and Genetic Algorithm, Knowledge-Based Systems 43 (2013) 30-42. 
[39] J. J. Rodríguez and L. I. Kuncheva, Rotation Forest: A New Classifier Ensemble Method, IEEE Transactions on Pattern Analysis and Machine Intelligence, 28 (10) (2006) 1619-1630.

[40] S. Rosset, Z. Ji and T. Hastie, Boosting as a regularized path to a maximum margin classifier, Journal of Machine learning research, 5 (2004) 941-973.

[41] R. E. Schapire, The strength of weak learnability, Machine Learning, 5 (1990) 197-227.

[42] R. E. Schapire, Y. Freund, P. Bartlett and W. S. Lee, Boosting the margin: A new explanation for the effectiveness of voting methods, The Annals of Statistics, 26 (5) (1998) 1651-1686.

[43] J. Shawe-Taylor, P. L. Bartlett, R. C. Williamson and M. Anthony, A framework for structural risk minimisation, In Proceedings of the Ninth Annual Conference on Computational Learning Theory, pp. 68-76, 1996.

[44] J. Shawe-Taylor and N. Cristianini, Margin Distribution Bounds on Generalization, EuroCOLT 1999, pp. 263-273.

[45] C. H. Shen and H. X. Li, Boosting Through Optimization of Margin Distributions, IEEE Transactions on Neural Networks, vol. 21 (4) (2010) 659-666.

[46] E. K. Tang, P. N. Suganthan and X. Yao, An analysis of diversity measures, Machine Learning, 65 (2006) 247-271.

[47] R. Tibshirani, Regression shrinkage and selection via the lasso, J. Royal. Statist. Soc B. 58 (1) (1996) 267-288.

[48] N. Ueda, Optimal linear combination of neural networks for improving classification performance, IEEE Transactions on Pattern Analysis and Machine Intelligence, 22 (2) (2000) 207-215.

[49] V. N. Vapnik, Estimation of Dependences Based on Empirical Data, Springer-Verlag, New York, 1982.

[50] K. Weinberger, J. Blitzer and L. Saul, Distance metric learning for large margin nearest neighbor classification, Advances in Neural Information Processing Systems (NIPS), 18 (2006) 1473-1480.

[51] L. W. Wang, M. Sugiyama, Z. X. Jing, C. Yang, Z. H. Zhou and J. F. Feng, A Refined Margin Analysis for Boosting Algorithms via Equilibrium Margin, Journal of Machine Learning Research, 12 (2011) 1835-1863.

[52] F. Yang, W. H. Lu, L. K. Luo and T. Li, Margin optimization based pruning for random forest, Neurocomputing, 94 (2012) 54-63.

[53] X. Yao and Y. Liu, Making use of population information in evolutionary artificial neural networks, IEEE Transactions on Systems, Man and Cybernetics, Part-B: Cybernetics, 28 (3) (1998) 417-425. 
[54] Y. Zhang, S. Burer and W. N. Street, Ensemble pruning via semi-definite programming, Journal of Machine Learning Research, 7 (2006) 1315-1338.

[55] Z. H. Zhou, J.X. Wu and W. Tang, Ensembling neural networks: many could be better than all, Artificial Intelligence, 137 (1-2) (2002) 239-263.

[56] Z. H. Zhou and Y. Yu, Ensembling Local Learners Through Multimodal Perturbation, IEEE Transactions on Systems, Man and Cybernetics, PartB: Cybernetics, 35 (4) (2005) 725-735.

[57] L. Zhang and W. D. Zhou, Sparse ensembles using weighted combination methods based on linear programming, Pattern Recognition, 44 (2011) 97106.

[58] L. Zhang and W. D. Zhou, On the sparseness of 1-norm support vector machines, Neural Networks, 23 (3) (2010) 373-385.

[59] X. Q. Zhu, P. Zhang, X. D. Lin and Y. Shi, Active Learning From Stream Data Using Optimal Weight Classifier Ensemble, IEEE Transactions on Systems, Man and Cybernetics, Part-B: Cybernetics, 40 (6) (2010) 16071621. 